Research Article

\title{
Computing Analysis for First Zagreb Connection Index and Coindex of Resultant Graphs
}

\author{
Muhammad Javaid (iD, ${ }^{1}$ Usman Ali, ${ }^{1}$ and Jia-Bao Liu $\mathbb{D I}^{2}$ \\ ${ }^{1}$ Department of Mathematics, School of Science, University of Management and Technology, Lahore 54770, Pakistan \\ ${ }^{2}$ School of Mathematics and Physics, Anhui Jianzhu University, Hefei 230601, China
}

Correspondence should be addressed to Muhammad Javaid; javaidmath@gmail.com

Received 20 August 2020; Revised 2 January 2021; Accepted 13 January 2021; Published 1 March 2021

Academic Editor: Yong Zhang

Copyright ( $) 2021$ Muhammad Javaid et al. This is an open access article distributed under the Creative Commons Attribution License, which permits unrestricted use, distribution, and reproduction in any medium, provided the original work is properly cited.

\begin{abstract}
A numeric parameter which studies the behaviour, structural, toxicological, experimental, and physicochemical properties of chemical compounds under several graphs' isomorphism is known as topological index. In 2018, Ali and Trinajstić studied the first Zagreb connection index $\left(Z C_{1}\right)$ to evaluate the value of a molecule. This concept was first studied by Gutman and Trinajstić in 1972 to find the solution of $\pi$-electron energy of alternant hydrocarbons. In this paper, the first Zagreb connection index and coindex are obtained in the form of exact formulae and upper bounds for the resultant graphs in terms of different indices of their factor graphs, where the resultant graphs are obtained by the product-related operations on graphs such as tensor product, strong product, symmetric difference, and disjunction. At the end, an analysis of the obtained results for the first Zagreb connection index and coindex on the aforesaid resultant graphs is interpreted with the help of numerical values and graphical depictions.
\end{abstract}

\section{Introduction}

Topological indices (TIs) are used in the study of cheminformatics which has key role in the formational structure of quantitative structures' activity and property relationships to examine the chemical reactivity and experimental activity of a chemical compound in a molecular graph [1]. So, TIs predict both physical and chemical features that are defined in the molecular graphs such as surface tension, solubility, connectivity, freezing point, boiling point, melting point, critical temperature, polarizability, heat of evaporation, and formation [2]. In addition, the medical behaviours and a number of drug particles of different compounds have formed with the help of various TIs in the pharmaceutical networks (see [3]). In particular, the TIs called by connection number-based Zagreb indices are used to compute the correlation values among various octane isomers such as heat of evaporation, acentric factor, molecular weight, connectivity, critical temperature, stability, and density (see $[4,5])$.

Product graphs play an essential part to develop new molecular graphs from simple graphs. For this purpose,
Ashrafi et al. [6] defined the concept of coindices for several products on graphs. Das et al. [7] computed upper bounds for multiplicative Zagreb indices of operations such as join, corona, Cartesian, disjunction, and composition. The reformulated, multiplicative, hyper, first, second, and third Zagreb coindices with certain properties are defined in [8-13]. Relations between Zagreb coindices and some distance-based indices are computed in [14]. For more details, see [15-18]. For this purpose, we can define some operations such as tensor product, strong product, symmetric difference, and disjunction in the following definitions.

Definition 1. Tensor (or Kronecker or conjunctive or direct) product of two graphs $G_{1}$ and $G_{2}$ is a graph $G_{1} \oslash G_{2}$ with vertex set $V\left(G_{1} \oslash G_{2}\right)=V\left(G_{1}\right) \oslash V\left(G_{2}\right)$ and edge set $E\left(G_{1} \oslash G_{2}\right)=\left\{\left(a_{1}, b_{1}\right)\left(a_{2}, b_{2}\right)\right.$, where $\left(a_{1}, b_{1}\right),\left(a_{2}, b_{2}\right) \in$ $\left.V\left(G_{1}\right) \oslash V\left(G_{2}\right)\right\}$ and

$$
\left[a_{1} a_{2} \in E\left(G_{1}\right)\right] \wedge\left[b_{1} b_{2} \in E\left(G_{2}\right)\right] .
$$


The connection number of a vertex $(a, b)$ of $G_{1} \oslash G_{2}$ is defined by $\tau_{Q_{1} \oslash Q_{2}}(a, b)=\tau_{G_{1}}(a)+\tau_{G_{2}}(b)+\tau_{G_{1}}(a) \tau_{G_{2}}(b)$. For more details, see Figure 1.

Definition 2. Strong product or normal product $\left(G_{1} \otimes G_{2}\right)$ of two graphs $G_{1}$ and $G_{2}$ is obtained by taking the vertex set and edge set as $V\left(Q_{1} \otimes Q_{2}\right)=V\left(Q_{1}\right) \otimes V\left(Q_{2}\right)$ and $E\left(Q_{1} \otimes Q_{2}\right)=\left\{\left(a_{1}, b_{1}\right)\left(a_{2}, b_{2}\right), \quad\right.$ where $\left(a_{1}, b_{1}\right),\left(a_{2}, b_{2}\right) \in$ $\left.V\left(Q_{1}\right) \otimes V\left(Q_{2}\right)\right\}$ with condition

$$
\begin{aligned}
& \text { either }\left[a_{1}=a_{2} \in V\left(G_{1}\right) \wedge b_{1} b_{2} \in E\left(Q_{2}\right)\right] \\
& \text { or }\left[b_{1}=b_{2} \in V\left(Q_{2}\right) \wedge a_{1} a_{2} \in E\left(Q_{1}\right)\right] \\
& \text { or }\left[a_{1} a_{2} \in E\left(Q_{1}\right)\right] \wedge\left[b_{1} b_{2} \in E\left(Q_{2}\right)\right] .
\end{aligned}
$$

The connection number of a vertex $(a, b)$ of $Q_{1} \otimes Q_{2}$ is defined by

$$
\begin{aligned}
\tau_{G_{1} \otimes G_{2}}(a, b)= & \tau_{G_{1}}(a)+\tau_{G_{2}}(b)+d G_{1}(a) \tau_{G_{1}}(a) \\
& +d_{G_{2}}(b) \tau_{G_{2}}(b)+\tau_{G_{1}}(a) \tau_{G_{2}}(b) \\
= & \tau_{G_{1}}(a)\left[1+d_{G_{1}}(a)\right] \\
& +\tau_{G_{2}}(b)\left[1+d_{G_{2}}(b)+\tau_{G_{1}}(a)\right],
\end{aligned}
$$

if $n_{1}, n_{2} \geq 4$. For more details, see Figure 2 .

Definition 3. Symmetric difference of two graphs $Q_{1}$ and $Q_{2}$ is a graph $G_{1} \ominus G_{2}$ with vertex set $V\left(Q_{1} \ominus Q_{2}\right)=V\left(Q_{1}\right) \ominus V\left(Q_{2}\right)$ and edge set $E\left(Q_{1} \ominus Q_{2}\right)=\left\{\left(a_{1}, b_{1}\right)\left(a_{2}, b_{2}\right)\right.$, where $\left(a_{1}, b_{1}\right)$, $\left.\left(a_{2}, b_{2}\right) \in V\left(Q_{1}\right) \ominus V\left(Q_{2}\right)\right\}$ and

$$
\begin{aligned}
& {\left[a_{1} a_{2} \in E\left(Q_{1}\right)\right]} \\
& \quad \text { or }\left[b_{1} b_{2} \in E\left(Q_{2}\right)\right],
\end{aligned}
$$

but not both hold at the same time, respectively.

The connection number of a vertex $(a, b)$ of $G_{1} \ominus G_{2}$ is defined by

$$
\begin{aligned}
\tau_{G_{1} \ominus G_{2}}(a, b)= & n_{1} n_{2}-1-n_{2} d_{G_{1}}(a)-n_{1} d_{G_{2}}(b) \\
& +2 d_{G_{1}}(a) d_{G_{2}}(b) \\
= & n_{2}\left[n_{1}-d_{G_{1}}(a)\right]+d_{G_{2}}(b)\left[2 d_{G_{1}}(a)-n_{1}\right]-1 .
\end{aligned}
$$

For more details, see Figure 3.

Definition 4. Disjunction of two graphs $G_{1}$ and $G_{2}$ is a graph $G_{1} \oplus G_{2}$ with vertex set $V\left(Q_{1} \oplus Q_{2}\right)=V\left(Q_{1}\right) \oplus V\left(Q_{2}\right)$ and edge set $E\left(Q_{1} \oplus Q_{2}\right)=\left\{\left(a_{1}, b_{1}\right)\left(a_{2}, b_{2}\right)\right.$, where $\left(a_{1}, b_{1}\right),\left(a_{2}, b_{2}\right) \in$ $\left.V\left(Q_{1}\right) \oplus V\left(Q_{2}\right)\right\}$ and

$$
\begin{aligned}
& {\left[a_{1} a_{2} \in E\left(Q_{1}\right)\right]} \\
& \quad \text { or }\left[b_{1} b_{2} \in E\left(Q_{2}\right)\right] .
\end{aligned}
$$

The connection number of a vertex $(a, b)$ of $G_{1} \oplus G_{2}$ is defined by

$$
\begin{aligned}
\tau_{G_{1} \oplus G_{2}}(a, b)= & n_{1} n_{2}-1-n_{2} d_{G_{1}}(a)-n_{1} d_{G_{2}}(b) \\
& +d_{G_{1}}(a) d_{G_{2}}(b) \\
= & n_{2}\left[n_{1}-d_{G_{1}}(a)\right]+d_{G_{2}}(b)\left[d_{G_{1}}(a)-n_{1}\right]-1 .
\end{aligned}
$$

For more details, see Figure 4.

The graph theory provides the significant tools in the field of modern chemistry that is exploited to develop several types of molecular graphs and also predicts their chemical properties. In 1972, Gutman and Trinajstić [19] defined the first degree-based (number of vertices at distance one) TI called the first Zagreb index to compute the total $\pi$-electron energy of the molecules in molecular graphs. There are several TIs in literature, but degree-based TIs are studied more than others (see [20-29]).

Ali and Trinajstić [30] restudied the concept of connection number-based (number of vertices at distance two) TIs that were also defined by Gutman and Trinajstić in the same paper in 1972 to compute the total electron energy of the alternant hydrocarbons. They recalled them as Zagreb connection indices and reported that the chemical capability of the Zagreb connection indices is better than the classical Zagreb indices for the thirteen physicochemical properties of octane isomers. After two years, a few works have delivered on these connection number-based descriptors. Ali et al. [31] computed the analysis of Zagreb connection indices and coindices for some chemical structures of operations on graphs. For further studies and properties of the Zagreb connection indices, we refer to [32-40]. Recently, Gong et al. [41, 42] developed blocking lot-streaming flow shop scheduling problems and dynamic interval multiobjective optimization problems. These problems have been considered in various studies which have a close relation with the topic considered in this paper. For more details, see $[43,44]$.

In this paper, we compute the analysis for the first Zagreb connection index and coindex of the resultant graphs in the shape of exact formulae and upper bounds in terms of their factor graphs, where resultant graphs are obtained by operations such as tensor product, strong product, symmetric difference, and disjunction. Moreover, at the end, an analysis of the first Zagreb connection index and coindex on the aforesaid operations is included with the help of their numerical values and graphical depictions.

The rest of the paper is as follows: Section 2 represents the preliminary definitions and key results which are used in the main results, Section 3 contains the main results of product on graphs, and Section 4 includes the analysis and conclusions.

\section{Preliminaries}

Let $G=(V(G), E(G))$ be a simple graph such that the order and size of $G$ are $|V(G)|=n$ and $|E(G)|=e$. The distance $d(a, b)$ between any two vertices $a$ and $b$ of a graph $G$ is equal to the length of a shortest path connecting them. For $b \in V(G)$ and a positive integer $p, N_{p}(b / G)=$ 

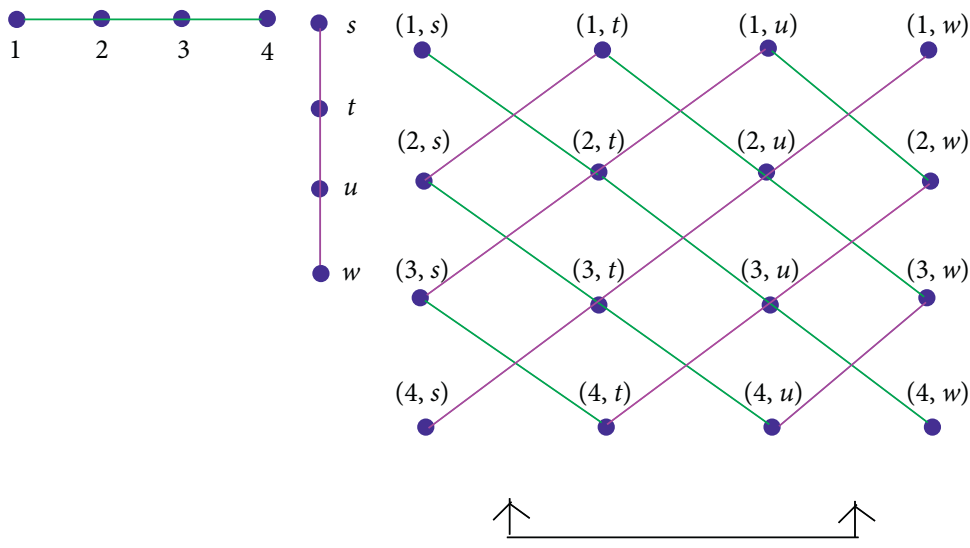

(a)

(b)

(c)

Figure 1: (a) $G_{1} \cong P_{4}$, (b) $G_{2} \cong P_{4}$, and (c) tensor product $\left(P_{4} \oslash P_{4}\right)$.

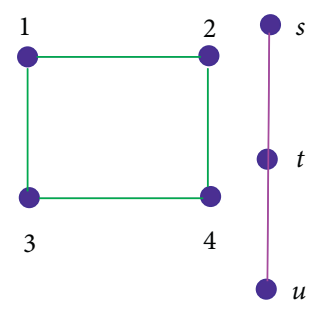

$(3, s)$

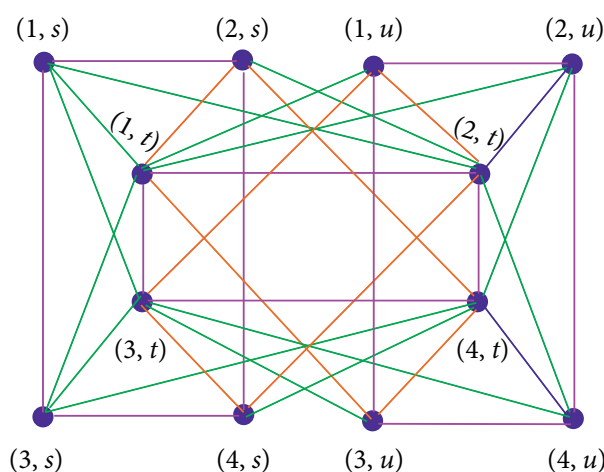

(c)

FIgURE 2: (a) $G_{1} \cong C_{4}$ (b) $G_{2} \cong P_{3}$, and (c) strong product $\left(C_{4} \otimes P_{3}\right)$.

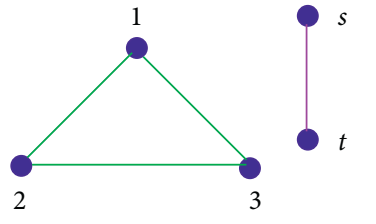

(a) (b)

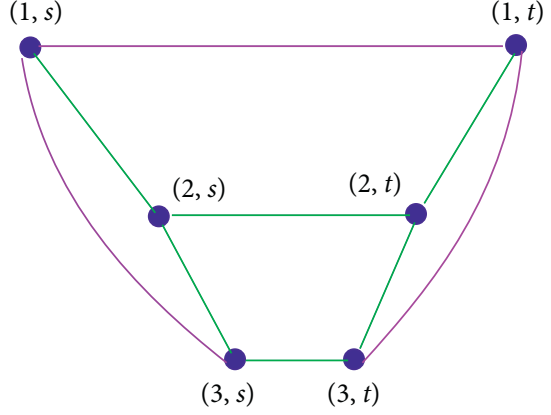

(c)

Figure 3: (a) $G_{1} \cong C_{3}$, (b) $G_{2} \cong P_{2}$, and (c) symmetric difference $\left(C_{3} \ominus P_{2}\right)$.

$\{u \in V(G): d(a, b)=p\}$ denotes the open $p$-neighborhood of $b$ in a graph $G$, where $d_{p}(b / G)=\left|N_{p}(b / G)\right|$ is called $p$-distance degree of a vertex $b$ in any graph $G$. The degree of a vertex $b$ in a graph $G$ is the number of edges incident on it, and it is denoted by $d_{G}(b)$. In particular:

If $p=1, d_{1}(b / G)=\left|N_{1}(b / G)\right|=d_{G}(b)=$ degree of $b$ (number of vertices at distance one from $b$ )
If $p=2, \quad d_{2}(b / G)=\left|N_{2}(b / G)\right|=\tau_{G}(b)=$ connection number of $b$ (number of vertices at distance two from $b$ )

The complement of a graph $G$ is denoted by $\bar{G}$. It is also simple with the same vertex set as of $G$, but edge set is defined as $E(\bar{G})=\{a b: a, b \in V(G) \wedge a b \notin E(G)\}$. Thus, $E(G) \cup E(\bar{G})=E\left(K_{n}\right)$, where $K_{n}$ is a complete graph of 

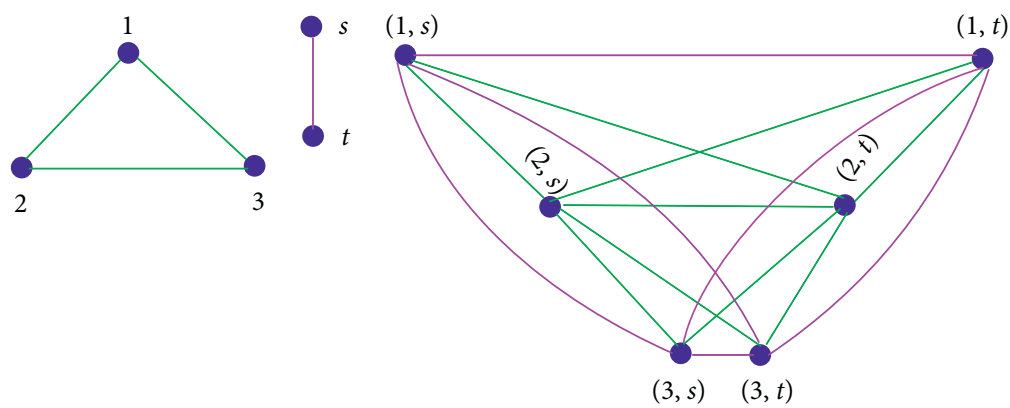

(a)

(b)

(c)

Figure 4 : (a) $G_{1} \cong C_{3}$, (b) $G_{2} \cong P_{2}$, and (c) disjunction $\left(C_{3} \cdot P_{2}\right.$ ).

order $n$ and size $\left|E\left(K_{n}\right)\right|=\left(\begin{array}{l}n \\ 2\end{array}\right)$. Moreover, if $|E(G)|=e$, then $|E(\bar{G})|=\left(\begin{array}{l}n \\ 2\end{array}\right)-e=\mu$ and $d_{\bar{G}}(b)=n-1-d_{G}(b)$, where $d_{G}(b)$ and $d_{\bar{G}}(b)$ are the degrees of the vertex $b$ in $G$ and $\bar{G}$, respectively. Now, throughout the paper, for two graphs $G_{1}$ and $G_{2}$, we assume that $\left|V\left(G_{1}\right)\right|=n_{1}$, $\left|V\left(G_{2}\right)\right|=n_{2},\left|E\left(G_{1}\right)\right|=e_{1}$, and $\left|E\left(G_{2}\right)\right|=e_{2}$. Finally, it is important to note that Zagreb connection coindices of $G$ are not Zagreb connection indices of $\bar{G}$ because the connection number works according to $G$. For any terminology or notion which are not mentioned here, we refer to $[45,46]$.

Definition 5. For a graph $G$, the first Zagreb index $\left(M_{1}(G)\right)$ and second Zagreb index $\left(M_{2}(G)\right)$ are defined as

$$
\begin{aligned}
& M_{1}(G)=\sum_{a b \in E(G)}\left[d_{G}(a)+d_{G}(b)\right], \\
& M_{2}(G)=\sum_{a b \in E(G)}\left[d_{G}(a) \times d_{G}(b)\right] .
\end{aligned}
$$

These degree-based indices are defined by Gutman, Trinajstić, and Ruscic (see $[19,47]$ ) which are frequently used to predict better outcomes of the various parameters related to the molecular networks such as chirality, complexity, entropy, heat energy, ZE-isomerism, heat capacity, absolute value of correlation coefficient, chromatographic, retention times in chromatographic, $\mathrm{pH}$, and molar ratio $[19,48]$. Symmetrical to these degree-based TIs, the connection-based TIs are discussed in Definitions 6 and 7.

Definition 6. For a graph $G$, the first Zagreb connection index $\left(Z C_{1}(G)\right)$ is defined as

$$
Z C_{1}(G)=\sum_{b \in V(G)}\left[\tau_{G}(b)\right]^{2} .
$$

Definition 7. For a graph $G$, the modified first Zagreb connection index $\left(Z C_{1}^{*}(G)\right)$ and second Zagreb connection index $\left(Z C_{2}(G)\right)$ are defined as

$$
\begin{aligned}
& Z C_{1}^{*}(G)=\sum_{a b \in E(G)}\left[\tau_{G}(a)+\tau_{G}(b)\right], \\
& Z C_{2}(G)=\sum_{a b \in E(G)}\left[\tau_{G}(a) \times \tau_{G}(b)\right] .
\end{aligned}
$$

Definition 8. For a graph $G$, the first Zagreb coindex $\left(\bar{M}_{1}(G)\right)$ and second Zagreb coindex $\left(\bar{M}_{2}(G)\right)$ are defined as

$$
\begin{aligned}
& \bar{M}_{1}(G)=\sum_{a b \notin E(G)}\left[d_{G}(a)+d_{G}(b)\right], \\
& \bar{M}_{2}(G)=\sum_{a b \notin E(G)}\left[d_{G}(a) \times d_{G}(b)\right] .
\end{aligned}
$$

These coindices associated with the degree-based classical Zagreb indices are defined by Ashrafi et al. (see [6]). The coindices associated with the connection-based Zagreb indices are defined in Definition 9.

Definition 9. For a graph $G$, the first Zagreb connection coindex $\left(\bar{Z} C_{1}(G)\right)$ and second Zagreb connection coindex $\left(\bar{Z} C_{2}(G)\right)$ are defined as

$$
\begin{aligned}
\bar{Z} C_{1}(G) & =\sum_{a b \notin E(G)}\left[\tau_{G}(a)+\tau_{G}(b)\right], \\
\bar{Z} C_{2}(G) & =\sum_{a b \notin E(G)}\left[\tau_{G}(a) \times \tau_{G}(b)\right] .
\end{aligned}
$$

The modified coindices associated with the connection number-based Zagreb indices are defined in Definition 10. These modified coindices associated with the connection number-based Zagreb indices are defined by Ali et al. (see [49]).

Definition 10. For a graph $G$, the modified first Zagreb connection coindex $\left(M \bar{Z} C_{1}(G)\right)$ and the modified second Zagreb connection coindex $\left(M \bar{Z} C_{2}(G)\right)$ are defined as 


$$
\begin{aligned}
& M \bar{Z} C_{1}(K)=\sum_{u v \notin E(K)}\left[d_{K}(u) \tau_{K}(v)+d_{K}(v) \tau_{K}(u)\right], \\
& M \bar{Z} C_{2}(K)=\sum_{u v \notin E(K)}\left[d_{K}(u) \tau_{K}(u)+d_{K}(v) \tau_{K}(v)\right] .
\end{aligned}
$$

The degree/connection-based coindices defined in Definitions 8-10 study the various physicochemical and isomer properties of molecules on the basis of the adjacency and nonadjacency pairs of vertices in the molecular networks. For more details, see $[6,30,31,36]$.

Now, we present some important results which are used in the main results.

Lemma 1 (see [50]). Let $G$ be a connected graph and $\bar{G}$ be its complement with $n$ vertices and e edges. Then,

(a) $\sum_{b \in V(G)} d_{G}(b)=2 e$

(b) $M_{1}(\bar{G}) \geq M_{1}(G)-4$
Lemma 2 (see [36]). Let $G$ be a connected and $\left\{C_{3}, C_{4}\right\}$-free graph with $n$ vertices and e edges. Then,

(a) $\sum_{b \in V\left(G_{1}\right)} \tau_{G_{1}}(b)=M_{1}\left(G_{1}\right)-2 e_{1}=A$

(b) $\sum_{b \in V\left(G_{2}\right)} \tau_{G_{2}}(b)=M_{1}\left(G_{2}\right)-2 e_{2}=B$

\section{Main Results}

This section contains the main results for the first Zagreb connection index $\left(Z C_{1}\right)$ and first Zagreb connection coindex $\left(\bar{Z} C_{1}\right)$ of the product on graphs obtained under the operations of tensor product, strong product, symmetric difference, and disjunction.

Theorem 1. Let $G_{1}$ and $G_{2}$ be two connected and $\left\{C_{3}, C_{4}\right\}$-free graphs. Then, $Z C_{1}$ and $\bar{Z} C_{1}$ of the tensor product $\left(G_{1} \oslash G_{2}\right)$ are

$$
\begin{aligned}
& Z C_{1}\left(G_{1} \oslash G_{2}\right)=n_{2} Z C_{1}\left(G_{1}\right)+n_{1} Z C_{1}\left(G_{2}\right)+Z C_{1}\left(G_{1}\right) Z C_{1}\left(G_{2}\right)+2 A B+2 A Z C_{1}\left(G_{2}\right)+2 B Z C_{1}\left(G_{1}\right), \\
& \bar{Z} C_{1}\left(G_{1} \oslash G_{2}\right)=2 \mu_{2} \bar{Z} C_{1}\left(G_{1}\right)+2 \mu_{1} \bar{Z} C_{1}\left(G_{2}\right)+2 \sum_{a_{1} a_{2} \notin E\left(G_{1}\right)} \sum_{b_{1} b_{2} \notin E\left(G_{2}\right)}\left[\tau_{G_{1}}\left(a_{1}\right) \tau_{G_{2}}\left(b_{1}\right)+\tau_{G_{1}}\left(a_{2}\right) \tau_{G_{2}}\left(b_{2}\right)\right] .
\end{aligned}
$$

Proof. Since $\tau_{\mathrm{Q}_{1} \oslash Q_{2}}(a, b)=\tau_{G_{1}}(a)+\tau_{G_{2}}(b)+\tau_{G_{1}}(a) \tau_{G_{2}}(b)$, where $a \in V\left(G_{1}\right), b \in V\left(G_{2}\right)$, and $(a, b) \in G_{1} \oslash G_{2}$,

$$
\begin{aligned}
Z C_{1}\left(G_{1} \oslash G_{2}\right)= & \sum_{(a, b) \in V\left(G_{1} \oslash G_{2}\right)}\left[\tau_{G_{1} \oslash G_{2}}(a, b)\right]^{2} \\
= & \sum_{a \in V\left(G_{1}\right)} \sum_{b \in V\left(G_{2}\right)}\left[\tau_{G_{1}}(a)+\tau_{G_{2}}(b)+\tau_{G_{1}}(a) \tau_{G_{2}}(b)\right]^{2} \\
= & \sum_{a \in V\left(G_{1}\right)} \sum_{b \in V\left(G_{2}\right)}^{2}\left[\tau_{G_{1}}^{2}(a)+\tau_{G_{2}}^{2}(b)+\tau_{G_{1}}^{2}(a) \tau_{G_{2}}^{2}(b)+2 \tau_{G_{1}}(a)+\tau_{G_{2}}(b)+2 \tau_{G_{1}}(a) \tau_{G_{2}}^{2}(b)+2 \tau_{G_{1}}^{2}(a) \tau_{G_{2}}(b)\right] \\
= & \sum_{a \in V\left(G_{1}\right)}\left[n_{2} \tau_{G_{1}}^{2}(a)+Z C_{1}\left(G_{2}\right)+\tau_{G_{1}}^{2}(a) Z C_{1}\left(G_{2}\right)+2 \tau_{G_{1}}(a)\left\{M_{1}\left(G_{2}\right)-2 e_{2}\right\}\right. \\
& \left.+2 \tau_{G_{1}}(a) Z C_{1}\left(G_{2}\right)+2 \tau_{G_{1}}^{2}(a)\left\{M_{1}\left(G_{2}\right)-2 e_{2}\right\}\right] .
\end{aligned}
$$

Using Lemma 2 (a) and (b),

$$
\begin{aligned}
Z C_{1}\left(G_{1} \oslash G_{2}\right)= & n_{2} Z C_{1}\left(G_{1}\right)+n_{1} Z C_{1}\left(G_{2}\right)+Z C_{1}\left(G_{1}\right) Z C_{1}\left(G_{2}\right)+2 A B+2 A Z C_{1}\left(G_{2}\right)+2 B Z C_{1}\left(G_{1}\right), \\
\bar{Z} C_{1}\left(G_{1} \oslash G_{2}\right)= & \sum_{\left(a_{1}, b_{1}\right)}\left[a_{2}, b_{2}\right) \notin E\left(\tau_{\left.G_{1} \oslash G_{2}\right)}\left(a_{1}, b_{1}\right)+\tau_{G_{1} \oslash G_{2}}\left(a_{2}, b_{2}\right)\right] \\
= & 2 \sum_{a_{1} a_{2} \notin E\left(G_{1}\right)} \sum_{b_{1} b_{2} \notin E\left(G_{2}\right)}\left[\left\{\tau_{G_{1}}\left(a_{1}\right)+\tau_{G_{2}}\left(b_{1}\right)+\tau_{G_{1}}\left(a_{1}\right) \tau_{G_{2}}\left(b_{1}\right)\right\}+\left\{\tau_{G_{1}}\left(a_{2}\right)+\tau_{G_{2}}\left(b_{2}\right)+\tau_{G_{1}}\left(a_{2}\right) \tau_{G_{2}}\left(b_{2}\right)\right\}\right] \\
= & 2 \sum_{a_{1} a_{2} \notin E\left(G_{1}\right)} \sum_{b_{1} b_{2} \notin E\left(G_{2}\right)}\left[\left\{\tau_{G_{1}}\left(a_{1}\right)+\tau_{G_{1}}\left(a_{2}\right)\right\}+\left\{\tau_{G_{2}}\left(b_{1}\right)+\tau_{G_{2}}\left(b_{2}\right)\right\}+\left\{\tau_{G_{1}}\left(a_{1}\right) \tau_{G_{2}}\left(b_{1}\right)+\tau_{G_{1}}\left(a_{2}\right) \tau_{G_{2}}\left(b_{2}\right)\right\}\right] \\
\bar{Z} C_{1}\left(G_{1} \oslash G_{2}\right) & =2 \mu_{2} \bar{Z} C_{1}\left(G_{1}\right)+2 \mu_{1} \bar{Z} C_{1}\left(G_{2}\right)+2 \sum_{a_{1} a_{2} \notin E\left(G_{1}\right)} \sum_{b_{1} b_{2} \notin E\left(G_{2}\right)}\left[\tau_{G_{1}}\left(a_{1}\right) \tau_{G_{2}}\left(b_{1}\right)+\tau_{G_{1}}\left(a_{2}\right) \tau_{G_{2}}\left(b_{2}\right)\right] .
\end{aligned}
$$


Theorem 2. Let $G_{1}$ and $G_{2}$ be two connected and $\left\{C_{3}, C_{4}\right\}$-free graphs. Then, $Z C_{1}$ and $\bar{Z} C_{1}$ of the strong product $\left(G_{1} \otimes G_{2}\right)$ are

$$
\begin{aligned}
Z C_{1}\left(G_{1} \otimes G_{2}\right)= & 2 B Z C_{1}^{*}\left(G_{1}\right)+2 A Z C_{1}^{*}\left(G_{2}\right)+2 Z C_{1}^{*}\left(G_{1}\right) Z C_{1}^{*}\left(G_{2}\right)+\left(n_{2}+2 B\right) Z C_{1}\left(G_{1}\right) \\
+ & \left(n_{1}+2 A\right) Z C_{1}\left(G_{2}\right)+Z C_{1}\left(G_{1}\right) Z C_{1}\left(G_{2}\right)+2 A B+2\left(n_{2}+B\right) \sum_{a \in V\left(G_{1}\right)} d_{G_{1}}(a) \tau_{G_{1}}^{2}(a)+2\left(n_{1}+A\right) \\
& \cdot \sum_{b \in V\left(G_{2}\right)} d_{G_{2}}(b) \tau_{G_{2}}^{2}(b)+n_{2} \sum_{a \in V\left(G_{1}\right)} d_{G_{1}}^{2}(a) \tau_{G_{1}}^{2}(a)+n_{1} \sum_{b \in V\left(G_{2}\right)} d_{G_{2}}^{2}(b) \tau_{G_{2}}^{2}(b),
\end{aligned}
$$

$$
\begin{aligned}
\bar{Z} C_{1}\left(G_{1} \otimes G_{2}\right) \leq & \left(n_{2}+2 \mu_{2}\right) M \bar{Z} C_{2}\left(G_{1}\right)+\left(n_{1}+2 \mu_{1}\right) M \bar{Z} C_{2}\left(G_{2}\right)+\left(n_{2}+2 \mu_{2}+B\right) \bar{Z} C_{1}\left(G_{1}\right) \\
& +\left(n_{1}+2 \mu_{1}+A\right) \bar{Z} C_{1}\left(G_{2}\right)+2\left[\mu_{2} Z C_{1}^{*}\left(G_{1}\right)+\mu_{1} Z C_{1}^{*}\left(G_{2}\right)\right]+2\left[\mu_{2} A+\mu_{1} B\right] \\
& +2 \sum_{\substack{a_{1} a_{2} \notin E\left(G_{1}\right) \\
a_{1} \neq a_{2} \wedge a_{1} \sim a_{2}}} \sum_{\substack{b_{1} b_{2} \notin E\left(G_{2}\right) \\
b_{1} \neq b_{2} \wedge b_{1} \sim b_{2}}}\left[\tau_{G_{1}}\left(a_{1}\right) \tau_{G_{2}}\left(b_{1}\right)+\tau_{G_{1}}\left(a_{2}\right) \tau_{G_{2}}\left(b_{2}\right)\right] .
\end{aligned}
$$

Proof. Since $\tau_{G_{1} \otimes G_{2}}(a, b)=\tau_{G_{1}}(a)\left[1+d_{G_{1}}(a)\right]+\tau_{G_{2}}(b)[1+$ $\left.d_{G_{2}}(b)+\tau_{G_{1}}(a)\right]$, where $a \in V\left(G_{1}\right), \quad b \in V\left(G_{2}\right)$, and $(a, b) \in G_{1} \otimes G_{2}$,

$$
\begin{aligned}
& Z C_{1}\left(G_{1} \otimes G_{2}\right)=\sum_{(a, b) \in V\left(G_{1} \otimes G_{2}\right)}\left[\tau_{G_{1} \otimes G_{2}}(a, b)\right]^{2} \\
& =\sum_{a \in V\left(G_{1}\right)} \sum_{b \in V\left(G_{2}\right)}\left[\tau_{G_{1}}(a)\left\{1+d_{G_{1}}(a)\right\}+\tau_{G_{2}}(b)\left\{1+d_{G_{2}}(b)+\tau_{G_{1}}(a)\right\}\right]^{2} \\
& =\sum_{a \in V\left(G_{1}\right)} \sum_{b \in V\left(G_{2}\right)}\left[\tau_{G_{1}}^{2}(a)\left\{1+d_{G_{1}}(a)\right\}^{2}+\tau_{G_{2}}^{2}(b)\left\{1+d_{G_{2}}(b)+\tau_{G_{1}}(a)\right\}^{2}+\right. \\
& \left.2 \tau_{G_{1}}(a) \tau_{G_{2}}(b)\left\{1+d_{G_{1}}(a)\right\}\left\{1+d_{G_{2}}(b)+\tau_{G_{1}}(a)\right\}\right] \\
& =\sum_{a \in V\left(G_{1}\right)} \sum_{b \in V\left(G_{2}\right)}\left[\tau_{G_{1}}^{2}(a)\left\{1+d_{G_{1}}^{2}(a)+2 d_{G_{1}}(a)\right\}+\tau_{G_{2}}^{2}(b)\left[\left\{1+d_{G_{2}}(b)\right\}^{2}+\tau_{G_{1}}^{2}(a)+2\left\{1+d_{G_{2}}(b)\right\} \tau_{G_{1}}(a)\right]\right. \\
& \left.+2 \tau_{G_{1}}(a) \tau_{G_{2}}(b)\left\{1+d_{G_{2}}(b)+\tau_{G_{1}}(a)+d_{G_{1}}(a)+d_{G_{1}}(a) d_{G_{2}}(b)+d_{G_{1}}(a) \tau_{G_{1}}(a)\right\}\right] \\
& =\sum_{a \in V\left(G_{1}\right)} \sum_{b \in V\left(G_{2}\right)}\left[\tau_{G_{1}}^{2}(a)+d_{G_{1}}^{2}(a) \tau_{G_{1}}^{2}(a)+2 d_{G_{1}}(a) \tau_{G_{1}}^{2}+\tau_{G_{2}}^{2}(b)+d_{G_{2}}^{2}(b) \tau_{G_{2}}^{2}(b)+d_{G_{2}}(b) \tau_{G_{2}}^{2}(b)\right. \\
& +\tau_{G_{1}}^{2}(a) \tau_{G_{2}}^{2}(b)+2 \tau_{G_{1}}(a) \tau_{G_{2}}^{2}(b)+2 d_{G_{2}}(b) \tau_{G_{1}}(a) \tau_{G_{2}}^{2}(b)+2 \tau_{G_{1}}(a) \tau_{G_{2}}(b)+2 d_{G_{2}}(b) \tau_{G_{1}}(a) \tau_{G_{2}}(b) \\
& \left.+2 \tau_{G_{1}}^{2}(a) \tau_{G_{2}}(b)+2 d_{G_{1}}(a) \tau_{G_{1}}(a) \tau_{G_{2}}(b)+2 d_{G_{1}}(a) \tau_{G_{1}}(a) d_{G_{2}}(b) \tau_{G_{2}}(b)+2 d_{G_{1}}(a) \tau_{G_{1}}^{2}(a) \tau_{G_{2}}(b)\right] .
\end{aligned}
$$


Using Lemma 2 (a) and (b),

$$
\begin{aligned}
Z C_{1}\left(G_{1} \otimes G_{2}\right)= & 2 B Z C_{1}^{*}\left(G_{1}\right)+2 A Z C_{1}^{*}\left(G_{2}\right) \\
& +2 Z C_{1}^{*}\left(G_{1}\right) Z C_{1}^{*}\left(G_{2}\right)+\left(n_{2}+2 B\right) Z C_{1}\left(G_{1}\right) \\
& +\left(n_{1}+2 A\right) Z C_{1}\left(G_{2}\right)+Z C_{1}\left(G_{1}\right) Z C_{1}\left(G_{2}\right) \\
& +2 A B+2\left(n_{2}+B\right) \sum_{a \in V\left(G_{1}\right)} d_{G_{1}}(a) \tau_{G_{1}}^{2}(a) \\
& +2\left(n_{1}+A\right) \sum_{b \in V\left(G_{2}\right)} d_{G_{2}}(b) \tau_{G_{2}}^{2}(b) \\
& +n_{2} \sum_{a \in V\left(G_{1}\right)} d_{G_{1}}^{2}(a) \tau_{G_{1}}^{2}(a)+n_{1} \sum_{b \in V\left(G_{2}\right)} d_{G_{2}}^{2}(b) \tau_{G_{2}}^{2}(b), \\
\bar{Z} C_{1}\left(G_{1} \otimes G_{2}\right)= & \left(\sum_{1}, b_{1}\right)\left(a_{2}, b_{2}\right) \notin E\left(G_{1} \otimes G_{2}\right) \\
= & \sum_{a \in V\left(G_{1}\right) b_{1} b_{2} \notin E\left(G_{2}\right)}\left[\tau_{G_{1} \otimes G_{2}}\left(a, b_{1}\right)+\tau_{G_{1} \otimes G_{2}}\left(a, b_{2}\right)\right] \\
& +\sum_{b \in V\left(G_{2}\right)} \sum_{a_{1} a_{2} \notin E\left(G_{1}\right)}\left[\tau_{G_{1} \otimes G_{2}}\left(a_{1}, b\right)+\tau_{G_{1} \otimes G_{2}}\left(a_{2}, b\right)\right] \\
& +\sum_{\substack{a_{1} a_{2} \notin E\left(G_{1}\right) \\
a_{1} \neq a_{2} \wedge a_{1} \sim a_{2}}}\left[\tau_{1} \neq b_{G_{1} \wedge b_{1} \sim b_{2}}\left(a_{G_{1} \otimes G_{2}}\left(a_{1}, b_{2}\right)+\tau_{G_{1}}\right)\right]
\end{aligned}
$$

We take

$$
\begin{aligned}
& \sum_{a \in V\left(G_{1}\right)} \sum_{b_{1} b_{2} \notin E\left(G_{2}\right)}\left[\tau_{G_{1} \otimes G_{2}}\left(a, b_{1}\right)+\tau_{G_{1} \otimes G_{2}}\left(a, b_{2}\right)\right] \\
& =\sum_{a \in V\left(G_{1}\right)} \sum_{b_{1} b_{2} \notin E\left(G_{2}\right)}\left[\left\{\tau_{G_{1}}(a)+\tau_{G_{2}}\left(b_{1}\right)+d_{G_{1}}(a) \tau_{G_{1}}(a)+d_{G_{2}}\left(b_{1}\right) \tau_{G_{2}}\left(b_{1}\right)+\tau_{G_{1}}(a) \tau_{G_{2}}\left(b_{1}\right)\right\}\right. \\
& \left.\left.\quad+\left\{\tau_{G_{1}}(a)+d_{\tau_{G_{2}}}\left(b_{2}\right) G_{1}(a) \tau_{G_{1}}(a)+d_{G_{2}}\left(b_{2}\right) \tau_{G_{2}}\left(b_{2}\right)+\tau_{G_{1}}(a) \tau_{G_{2}}\left(b_{2}\right)\right\}\right]\right] \\
& =\sum_{a \in V\left(G_{1}\right)} \sum_{b_{1} b_{2} \notin E\left(G_{2}\right)}\left[2 \tau_{G_{1}}(a)+\left\{\tau_{G_{2}}\left(b_{1}\right)+\tau_{G_{2}}\left(b_{2}\right)\right\}+2 d_{G_{1}}(a) \tau_{G_{1}}(a)+\left\{d_{G_{2}}\left(b_{1}\right) \tau_{G_{2}}\left(b_{1}\right)\right.\right. \\
& \left.\left.\quad+d_{G_{2}}\left(b_{2}\right) \tau_{G_{2}}\left(b_{2}\right)\right\}+\tau_{G_{1}}(a)\left\{\tau_{G_{2}}\left(b_{1}\right)+\tau_{G_{2}}\left(b_{2}\right)\right\}\right] .
\end{aligned}
$$

We know that

$$
\begin{aligned}
\sum_{b_{1} b_{2} \notin E\left(G_{2}\right)} & =\left(\begin{array}{c}
n_{2} \\
2
\end{array}\right)-e_{2}=\mu_{2} \\
& =2 \mu_{2} A+n_{1} \bar{Z} C_{1}\left(G_{2}\right)+2 \mu_{2} Z C_{1}^{*}\left(G_{1}\right)+n_{1} M \bar{Z} C_{2}\left(G_{2}\right)+A \bar{Z} C_{1}\left(G_{2}\right) .
\end{aligned}
$$

Also, we take

$$
\begin{aligned}
& \sum_{b \in V\left(G_{2}\right)} \sum_{a_{1} a_{2} \notin E\left(G_{1}\right)}\left[\tau_{G_{1} \otimes G_{2}}\left(a_{1}, b\right)+\tau_{G_{1} \otimes G_{2}}\left(a_{2}, b\right)\right] \\
& \sum_{b \in V\left(G_{2}\right)} \sum_{a_{1} a_{2} \notin E\left(G_{1}\right)}\left[\left\{\tau_{G_{1}}\left(a_{1}\right)+\tau_{G_{2}}(b)+d G_{1}\left(a_{1}\right) \tau_{G_{1}}\left(a_{1}\right)+d_{G_{2}}(b) \tau_{G_{2}}(b)+\tau_{G_{1}}\left(a_{1}\right) \tau_{G_{2}}(b)\right\}\right. \\
& \left.\quad+\left\{\tau_{G_{1}}\left(a_{2}\right)+\tau_{G_{2}}(b)+d G_{1}\left(a_{2}\right) \tau_{G_{1}}\left(a_{2}\right)+d_{G_{2}}(b) \tau_{G_{2}}(b)+\tau_{G_{1}}\left(a_{2}\right) \tau_{G_{2}}(b)\right\}\right] \\
& =n_{2} \bar{Z} C_{1}\left(G_{1}\right)+2 \mu_{1} B+n_{2} M \bar{Z} C_{2}\left(G_{1}\right)+2 \mu_{1} Z C_{1}^{*}\left(G_{2}\right)+B \bar{Z} C_{1}\left(G_{1}\right) .
\end{aligned}
$$


Again, we take

$$
\begin{aligned}
& \sum_{\substack{a_{1} a_{2} \notin E\left(G_{1}\right) \\
a_{1} \neq a_{2} \wedge a_{1} \sim a_{2}}} \sum_{\substack{b_{1} b_{2} \notin E\left(G_{2}\right) \\
b_{1} \neq b_{2} \wedge b_{1} \sim b_{2}}}\left[\tau_{G_{1} \otimes G_{2}}\left(a_{1}, b_{1}\right)+\tau_{G_{1} \otimes G_{2}}\left(a_{2}, b_{2}\right)\right] \\
& \leq 2 \sum_{\substack{a_{1} a_{2} \notin E\left(G_{1}\right) \\
a_{1} \neq a_{2} \wedge a_{1} \sim a_{2}}} \sum_{\substack{b_{1} b_{2} \notin E\left(G_{2}\right) \\
b_{1} \neq b_{2} \wedge b_{1} \sim b_{2}}}\left[\left\{\tau_{G_{1}}\left(a_{1}\right)+\tau_{G_{2}}\left(b_{1}\right)+d G_{1}\left(a_{1}\right) \tau_{G_{1}}\left(a_{1}\right)+d_{G_{2}}\left(b_{1}\right) \tau_{G_{2}}\left(b_{1}\right)+\tau_{G_{1}}\left(a_{1}\right) \tau_{G_{2}}\left(b_{1}\right)\right.\right. \\
& \left.+\left\{\tau_{G_{1}}\left(a_{2}\right)+\tau_{G_{2}}\left(b_{2}\right)+d G_{1}\left(a_{2}\right) \tau_{G_{1}}\left(a_{2}\right)+d_{G_{2}}\left(b_{2}\right) \tau_{G_{2}}\left(b_{2}\right)+\tau_{G_{1}}\left(a_{2}\right) \tau_{G_{2}}\left(b_{2}\right)\right\}\right]
\end{aligned}
$$

Similarly $=2 \mu_{2} \bar{Z} C_{1}\left(G_{1}\right)+2 \mu_{1} \bar{Z} C_{1}\left(G_{2}\right)+2 \mu_{2} M \bar{Z} C_{2}\left(G_{1}\right)+2 \mu_{1} M \bar{Z} C_{2}\left(G_{2}\right)$

$$
+2 \sum_{\substack{a_{1} a_{2} \notin E\left(G_{1}\right) \\ a_{1} \neq a_{2} \wedge a_{1} \sim a_{2}}} \sum_{\substack{b_{1} b_{2} \notin E\left(G_{2}\right) \\ b_{1} \neq b_{2} \wedge b_{1} \sim b_{2}}}\left[\tau_{G_{1}}\left(a_{1}\right) \tau_{G_{2}}\left(b_{1}\right)+\tau_{G_{1}}\left(a_{2}\right) \tau_{G_{2}}\left(b_{2}\right)\right] .
$$

Consequently,

$$
\begin{aligned}
\bar{Z} C_{1}\left(G_{1} \otimes G_{2}\right) \leq & \left(n_{2}+2 \mu_{2}\right) M \bar{Z} C_{2}\left(G_{1}\right)+\left(n_{1}+2 \mu_{1}\right) M \bar{Z} C_{2}\left(G_{2}\right)+\left(n_{2}+2 \mu_{2}+B\right) \bar{Z} C_{1}\left(G_{1}\right) \\
& +\left(n_{1}+2 \mu_{1}+A\right) \bar{Z} C_{1}\left(G_{2}\right)+2\left[\mu_{2} Z C_{1}^{*}\left(G_{1}\right)+\mu_{1} Z C_{1}^{*}\left(G_{2}\right)\right]+2\left[\mu_{2} A+\mu_{1} B\right] \\
& +2 \sum_{\substack{a_{1} a_{2} \notin E\left(G_{1}\right) \\
a_{1} \neq a_{2} \wedge a_{1} \sim a_{2}}} \sum_{\substack{b_{1} b_{2} \notin E\left(G_{2}\right) \\
b_{1} \neq b_{2} \wedge b_{1} \sim b_{2}}}\left[\tau_{G_{1}}\left(a_{1}\right) \tau_{G_{2}}\left(b_{1}\right)+\tau_{G_{1}}\left(a_{2}\right) \tau_{G_{2}}\left(b_{2}\right)\right] .
\end{aligned}
$$

Theorem 3. Let $G_{1}$ and $G_{2}$ be two connected and $\left\{C_{3}, C_{4}\right\}$-free graphs. Then, $Z C_{1}$ and $\bar{Z} C_{1}$ of the symmetric difference $\left(G_{1} \ominus G_{2}\right)$ are

$$
\begin{aligned}
Z C_{1}\left(G_{1} \ominus G_{2}\right)= & n_{2}\left(n_{2}^{2}-8 e_{2}\right) M_{1}\left(G_{1}\right)+n_{1}\left(n_{1}^{2}-8 e_{1}\right) M_{1}\left(G_{2}\right)+4 M_{1}\left(G_{1}\right) M_{1}\left(G_{2}\right) \\
& +n_{1} n_{2}\left(n_{1}^{2} n_{2}^{2}-4 n_{2}^{2} e_{1}-4 n_{1}^{2} e_{2}-2 n_{1} n_{2}+24 e_{1} e_{2}+1\right)+4\left(n_{1}^{2} e_{2}+n_{2}^{2} e_{1}-4 e_{1} e_{2}\right), \\
\bar{Z} C_{1}\left(G_{1} \ominus G_{2}\right)= & \left(4 e_{2}-n_{2}^{2}\right) \bar{M}_{1}\left(G_{1}\right)+\left(4 e_{1}-n_{1}^{2}\right) \bar{M}_{1}\left(G_{2}\right)+2 \mu_{1}\left(n_{1} n_{2}^{2}-n_{2}-2 n_{1} e_{2}\right) \\
& +2 \mu_{2}\left(n_{1}^{2} n_{2}-n_{1}-2 n_{2} e_{1}\right) .
\end{aligned}
$$

Proof. Since $\tau_{G_{1} \ominus G_{2}}(a, b)=n_{2}\left[n_{1}-d_{G_{1}}(a)\right]+d_{G_{2}}(b) \quad\left[2 d_{G_{1}}\right.$ (a) $\left.-n_{1}\right]-1$, where $a \in V\left(G_{1}\right), \quad b \in V\left(G_{2}\right)$, and $(a, b)$ $\in G_{1} \ominus G_{2}$, 


$$
\begin{aligned}
Z C_{1}\left(G_{1} \ominus G_{2}\right)= & \sum_{(a, b) \in V\left(G_{1} \ominus G_{2}\right)}\left[\tau_{G_{1} \ominus G_{2}}(a, b)\right]^{2} \\
= & \sum_{a \in V\left(G_{1}\right)} \sum_{b \in V\left(G_{2}\right)}\left[n_{2}\left\{n_{1}-d_{G_{1}}(a)\right\}+d_{G_{2}}(b)\left\{2 d_{G_{1}}(a)-n_{1}\right\}-1\right]^{2} \\
= & \sum_{a \in V\left(G_{1}\right)} \sum_{b \in V\left(G_{2}\right)}\left[n_{2}^{2}\left\{n_{1}-d_{G_{1}}(a)\right\}^{2}+d_{G_{2}}^{2}(b)\left\{2 d_{G_{1}}(a)-n_{1}\right\}^{2}+1+2 n_{2} d_{G_{2}}(b)\left\{n_{1}-d_{G_{1}}(a)\right\}\right. \\
& \left.\left\{2 d_{G_{1}}(a)-n_{1}\right\}-2 d_{G_{2}}(b)\left\{2 d_{G_{1}}(a)-n_{1}\right\}-2 n_{2}\left\{n_{1}-d_{G_{1}}(a)\right\}\right] \\
= & n_{1}^{3} n_{2}^{3}+n_{2}^{3} M_{1}\left(G_{1}\right)-4 n_{1} n_{2}^{3} e_{1}+4 M_{1}\left(G_{1}\right) M_{1}\left(G_{2}\right)+n_{1}^{3} M_{1}\left(G_{2}\right)-8 n_{1} e_{1} M_{1}\left(G_{2}\right)+n_{1} n_{2} \\
& +16 n_{1} n_{2} e_{1} e_{2}-4 n_{1}^{3} n_{2} e_{2}-8 n_{2} e_{2} M_{1}\left(G_{1}\right)+8 n_{1} n_{2} e_{1} e_{2}-16 e_{1} e_{2}+4 n_{1}^{2} e_{2}-2 n_{1}^{2} n_{2}^{2}+4 n_{2}^{2} e_{1}, \\
Z C_{1}\left(G_{1} \ominus G_{2}\right)= & n_{2}\left(n_{2}^{2}-8 e_{2}\right) M_{1}\left(G_{1}\right)+n_{1}\left(n_{1}^{2}-8 e_{1}\right) M_{1}\left(G_{2}\right)+4 M_{1}\left(G_{1}\right) M_{1}\left(G_{2}\right)+n_{1} n_{2} \\
& \left(n_{1}^{2} n_{2}^{2}-4 n_{2}^{2} e_{1}-4 n_{1}^{2} e_{2}-2 n_{1} n_{2}+24 e_{1} e_{2}+1\right)+4\left(n_{1}^{2} e_{2}+n_{2}^{2} e_{1}-4 e_{1} e_{2}\right) . \\
\bar{Z} C_{1}\left(G_{1} \ominus G_{2}\right)= & \\
& \left(a_{1}, b_{1}\right)\left(a_{2}, b_{2}\right) \notin E\left(G_{1} \ominus G_{2}\right) \\
= & \left.\sum_{a \in V\left(G_{1}\right) b_{1} b_{2} \notin E\left(G_{2}\right)}\left[\tau_{G_{1} \ominus G_{2}}\left(a_{1}, b_{1}\right)+\tau_{G_{1} \ominus G_{2}}\left(a, a_{2}, b_{2}\right)\right]+\tau_{G_{1} \ominus G_{2}}\left(a, b_{2}\right)\right]+\sum_{b \in V\left(G_{2}\right)} \sum_{a_{1} a_{2} \notin E\left(G_{1}\right)}\left[\tau_{G_{1} \ominus G_{2}}\left(a_{1}, b\right)+\tau_{G_{1} \ominus G_{2}}\left(a_{2}, b\right)\right] \\
& \cdot \sum_{a \in V\left(G_{1}\right) b_{1} b_{2} \notin E\left(G_{2}\right)}\left[\tau_{G_{1} \ominus G_{2}}\left(a, b_{1}\right)+\tau_{G_{1} \ominus G_{2}}\left(a, b_{2}\right)\right] \sum_{b \in V\left(G_{2}\right)} \sum_{a_{1} a_{2} \notin E\left(G_{1}\right)}\left[\tau_{G_{1} \ominus G_{2}}\left(a_{1}, b\right)+\tau_{G_{1} \ominus G_{2}}\left(a_{2}, b\right)\right] \\
& \cdot\left[\tau_{G_{1} \ominus G_{2}}\left(a_{1}, b_{1}\right)+\tau_{G_{1} \ominus G_{2}}\left(a_{2}, b_{2}\right)\right] .
\end{aligned}
$$

We take

$$
\begin{aligned}
& \sum_{a \in V\left(G_{1}\right)} \sum_{b_{1} b_{2} \notin E\left(G_{2}\right)}\left[\tau_{G_{1} \ominus G_{2}}\left(a, b_{1}\right)+\tau_{G_{1} \ominus G_{2}}\left(a, b_{2}\right)\right] \\
& =\sum_{a \in V\left(G_{1}\right)} \sum_{b_{1} b_{2} \notin E\left(G_{2}\right)}\left[\left\{n_{1} n_{2}-1-n_{2} d_{G_{1}}(a)-n_{1} d_{G_{2}}\left(b_{1}\right)+2 d_{G_{1}}(a) d_{G_{2}}\left(b_{1}\right)\right\}\right. \\
& \left.\quad+\left\{n_{1} n_{2}-1-n_{2} d_{G_{1}}(a)-n_{1} d_{G_{2}}\left(b_{2}\right)+2 d_{G_{1}}(a) d_{G_{2}}\left(b_{2}\right)\right\}\right] .
\end{aligned}
$$

Similarly $=2 n_{1}^{2} n_{2} \mu_{2}-2 n_{1} \mu_{2}-4 n_{2} e_{1} \mu_{2}-n_{1}^{2} \bar{M}_{1}\left(G_{2}\right)+4 e_{1} \bar{M}_{1}\left(G_{2}\right)$.

Also, we take

$$
\sum_{b \in V\left(G_{2}\right) a_{1} a_{2} \notin E\left(G_{1}\right)}\left[\tau_{G_{1} \ominus G_{2}}\left(a_{1}, b\right)+\tau_{G_{1} \ominus G_{2}}\left(a_{2}, b\right)\right] .
$$

Similarly $=2 n_{1} n_{2}^{2} \mu_{1}-2 n_{2} \mu_{1}-n_{2}^{2} \bar{M}_{1}\left(G_{1}\right)-4 n_{1} e_{2} \mu_{1}+4 e_{2} \bar{M}_{1}\left(G_{1}\right)$. 
Again, we take (null case)

$$
N=\sum_{\substack{a_{1} a_{2} \notin E\left(G_{1}\right) \\ a_{1} \neq a_{2} \wedge a_{1} \sim a_{2}}} \sum_{\substack{b_{1} b_{2} \notin E\left(G_{2}\right) \\ b_{1} \neq b_{2} \wedge b_{1} \uparrow b_{2}}}\left[\tau_{G_{1} \ominus G_{2}}\left(a_{1}, b_{1}\right)+\tau_{G_{1} \ominus G_{2}}\left(a_{2}, b_{2}\right)\right]=0 .
$$

We further take (also null case)

$$
N=\sum_{\substack{b_{1} b_{2} \notin E\left(G_{1}\right) \\ b_{1} \neq b_{2} \wedge b_{1} \sim b_{2}}} \sum_{\substack{a_{1} a_{2} \notin E\left(G_{2}\right) \\ a_{1} \neq a_{2} \wedge a_{1} \uparrow a_{2}}}\left[\tau_{G_{1} \ominus G_{2}}\left(a_{1}, b_{1}\right)+\tau_{G_{1} \ominus G_{2}}\left(a_{2}, b_{2}\right)\right]=0 .
$$

Consequently,

$$
\begin{aligned}
\bar{Z} C_{1}\left(G_{1} \ominus G_{2}\right)= & \left(4 e_{2}-n_{2}^{2}\right) \bar{M}_{1}\left(G_{1}\right)+\left(4 e_{1}-n_{1}^{2}\right) \bar{M}_{1}\left(G_{2}\right) \\
& +2 \mu_{1}\left(n_{1} n_{2}^{2}-n_{2}-2 n_{1} e_{2}\right) \\
& +2 \mu_{2}\left(n_{1}^{2} n_{2}-n_{1}-2 n_{2} e_{1}\right) .
\end{aligned}
$$

Theorem 4. Let $G_{1}$ and $G_{2}$ be two connected and $\left\{C_{3}, C_{4}\right\}$-free graphs. Then, $Z C_{1}$ and $\bar{Z} C_{1}$ of the disjunction $\left(G_{1} \oplus G_{2}\right)$ are

$$
\begin{aligned}
& Z C_{1}\left(G_{1} \oplus G_{2}\right)=n_{2}\left(n_{2}^{2}-4 e_{2}\right) M_{1}\left(G_{1}\right)+n_{1}\left(n_{1}^{2}-4 e_{1}\right) M_{1}\left(G_{2}\right)+M_{1}\left(G_{1}\right) M_{1}\left(G_{2}\right)+n_{1} n_{2} \\
& \left(n_{1}^{2} n_{2}^{2}-4 n_{2}^{2} e_{1}-4 n_{1}^{2} e_{2}-2 n_{1} n_{2}+16 e_{1} e_{2}+1\right)+4\left(n_{1}^{2} e_{2}+n_{2}^{2} e_{1}-2 e_{1} e_{2}\right) \\
& \bar{Z} C_{1}\left(G_{1} \oplus G_{2}\right)=\left(2 e_{2}-n_{2}^{2}-2 n_{2} \mu_{2}\right) \bar{M}_{1}\left(G_{1}\right)+\left(2 e_{1}-n_{1}^{2}-2 n_{1} \mu_{1}\right) \bar{M}_{1}\left(G_{2}\right) \\
& +2 \mu_{1}\left(n_{1} n_{2}^{2}-n_{2}-2 n_{1} e_{2}\right)+2 \mu_{2}\left(n_{1}^{2} n_{2}-n_{1}-2 n_{2} e_{1}\right)+4 \mu_{1} \mu_{2}\left(n_{1} n_{2}-1\right) \\
& +2 \sum_{\substack{a_{1} a_{2} \notin E\left(G_{1}\right) \\
a_{1} \neq a_{2} \wedge a_{1} \sim a_{2}}} \sum_{\substack{b_{1} b_{2} \notin E\left(G_{2}\right) \\
b_{1} \neq b_{2} \wedge b_{1} \sim b_{2}}}\left[d_{G_{1}}\left(a_{1}\right) d_{G_{2}}\left(b_{1}\right)+d_{G_{1}}\left(a_{2}\right) d_{G_{2}}\left(b_{2}\right)\right] .
\end{aligned}
$$

Proof. Since $\tau_{G_{1} \oplus G_{2}}(a, b)=n_{2}\left[n_{1}-d_{G_{1}}(a)\right]+d_{G_{2}}(b)\left[d_{G_{1}}(a)\right.$

$\left.-n_{1}\right]-1$, where $a \in V\left(G_{1}\right), b \in V\left(G_{2}\right)$, and $(a, b) \in G_{1} \oplus G_{2}$,

$$
\begin{aligned}
Z C_{1}\left(G_{1} \oplus G_{2}\right) & =\sum_{(a, b) \in V\left(G_{1} \oplus G_{2}\right)}\left[\tau_{G_{1} \oplus G_{2}}(a, b)\right]^{2} \\
& =\sum_{a \in V\left(G_{1}\right)} \sum_{b \in V\left(G_{2}\right)}\left[n_{2}\left\{n_{1}-d_{G_{1}}(a)\right\}+d_{G_{2}}(b)\left\{d_{G_{1}}(a)-n_{1}\right\}-1\right]^{2}
\end{aligned}
$$


Similarly,

$$
\begin{aligned}
& Z C_{1}\left(G_{1} \oplus G_{2}\right)=n_{2}\left(n_{2}^{2}-4 e_{2}\right) M_{1}\left(G_{1}\right)+n_{1}\left(n_{1}^{2}-4 e_{1}\right) M_{1}\left(G_{2}\right)+M_{1}\left(G_{1}\right) M_{1}\left(G_{2}\right)+n_{1} n_{2} \\
& \cdot\left(n_{1}^{2} n_{2}^{2}-4 n_{2}^{2} e_{1}-4 n_{1}^{2} e_{2}-2 n_{1} n_{2}+16 e_{1} e_{2}+1\right)+4\left(n_{1}^{2} e_{2}+n_{2}^{2} e_{1}-2 e_{1} e_{2}\right) \text {. } \\
& \bar{Z} C_{1}\left(G_{1} \oplus G_{2}\right)=\sum_{\left(a_{1}, b_{1}\right)} \sum_{\left(a_{2}, b_{2}\right) \notin E\left(G_{1} \oplus G_{2}\right)}\left[\tau_{G_{1} \oplus G_{2}}\left(a_{1}, b_{1}\right)+\tau_{G_{1} \oplus G_{2}}\left(a_{2}, b_{2}\right)\right]
\end{aligned}
$$

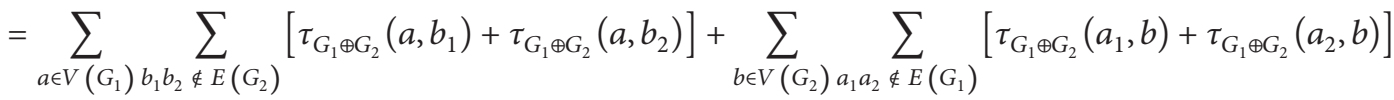

$$
\begin{aligned}
& \sum_{\substack{a_{1} a_{2} \notin E\left(G_{1}\right) \\
a_{1} \neq a_{2} \wedge a_{1} \sim a_{2}}} \sum_{\substack{b_{1} b_{2} \notin E\left(G_{2}\right) \\
b_{1} \neq b_{2} \wedge b_{1} \sim b_{2}}}\left[\tau_{G_{1} \oplus G_{2}}\left(a_{1}, b_{1}\right)+\tau_{G_{1} \oplus G_{2}}\left(a_{2}, b_{2}\right)\right] \\
& \sum_{a_{1} a_{2} \notin E\left(G_{1}\right)} \sum_{b_{1} b_{2} \notin E\left(G_{2}\right)}\left[\tau_{G_{1} \oplus G_{2}}\left(a_{1}, b_{1}\right)+\tau_{G_{1} \oplus G_{2}}\left(a_{2}, b_{2}\right)\right] \\
& a_{1} \neq a_{2} \wedge a_{1} \sim a_{2} \quad b_{1} \neq b_{2} \wedge b_{1} \uparrow b_{2} \\
& +\sum_{b_{1} b_{2} \notin E\left(G_{1}\right)} \sum_{a_{1} a_{2} \notin E\left(G_{2}\right)}\left[\tau_{G_{1} \ominus G_{2}}\left(a_{1}, b_{1}\right)+\tau_{G_{1} \ominus G_{2}}\left(a_{2}, b_{2}\right)\right] \text {. } \\
& b_{1} \neq b_{2} \wedge b_{1} \sim b_{2} \quad a_{1} \neq a_{2} \wedge a_{1} \uparrow a_{2}
\end{aligned}
$$

We take

$$
\begin{gathered}
\sum_{a \in V\left(G_{1}\right)} \sum_{b_{1} b_{2} \notin E\left(G_{2}\right)}\left[\tau_{G_{1} \oplus G_{2}}\left(a, b_{1}\right)+\tau_{G_{1} \oplus G_{2}}\left(a, b_{2}\right)\right] \\
=\sum_{a \in V\left(G_{1}\right)} \sum_{b_{1} b_{2} \notin E\left(G_{2}\right)}\left[\left\{n_{1} n_{2}-1-n_{2} d_{G_{1}}(a)-n_{1} d_{G_{2}}\left(b_{1}\right)+d_{G_{1}}(a) d_{G_{2}}\left(b_{1}\right)\right\}\right. \\
\left.+\left\{n_{1} n_{2}-1-n_{2} d_{G_{1}}(a)-n_{1} d_{G_{2}}\left(b_{2}\right)+d_{G_{1}}(a) d_{G_{2}}\left(b_{2}\right)\right\}\right] . \\
\text { Similarly }=2 n_{1}^{2} n_{2} \mu_{2}-2 n_{1} \mu_{2}-4 n_{2} e_{1} \mu_{2}-n_{1}^{2} \bar{M}_{1}\left(G_{2}\right)+2 e_{1} \bar{M}_{1}\left(G_{2}\right) .
\end{gathered}
$$

Also, we take

$$
\begin{gathered}
\sum_{\substack{a_{1} a_{2} \notin E\left(G_{1}\right) \\
a_{1} \neq a_{2} \wedge a_{1} \sim a_{2}}} \sum_{\substack{b_{1} b_{2} \notin E\left(G_{2}\right) \\
b_{1} \neq b_{2} \wedge b_{1} \sim b_{2}}}\left[\tau_{G_{1} \oplus G_{2}}\left(a_{1}, b_{1}\right)+\tau_{G_{1} \oplus G_{2}}\left(a_{2}, b_{2}\right)\right] \\
=2 \sum_{\substack{a_{1} a_{2} \notin E\left(G_{1}\right) \\
a_{1} \neq a_{2} \wedge a_{1} \sim a_{2} \text { b } \\
b_{1} \neq b_{2} \wedge b_{1} \sim b_{2}}}\left[\left\{n_{1} n_{2}-1-n_{2} d_{G_{1}}\left(a_{1}\right)-n_{1} d_{G_{2}}\left(b_{1}\right)+d_{G_{1}}\left(a_{1}\right) d_{G_{2}}\left(b_{1}\right)\right\}\right. \\
\left.+\left\{n_{1} n_{2}-1-n_{2} d_{G_{1}}\left(a_{2}\right)-n_{1} d_{G_{2}}\left(b_{2}\right)+d_{G_{1}}\left(a_{2}\right) d_{G_{2}}\left(b_{2}\right)\right\}\right] . \\
\text { Similarly }=4 n_{1} n_{2} \mu_{1} \mu_{2}-4 \mu_{1} \mu_{2}-2 n_{2} \mu_{2} \bar{M}_{1}\left(G_{1}\right)-2 n_{1} \mu_{1} \bar{M}_{1}\left(G_{2}\right) \\
+2 \sum_{\substack{a_{1} a_{2} \notin E\left(G_{1}\right) \\
a_{1} \neq a_{2} \wedge a_{1} \sim a_{2} \\
b_{1} \neq b_{1} \wedge b_{2} \wedge b_{1} \sim b_{2}}}\left[d_{G_{1}}\left(a_{1}\right) d_{G_{2}}\left(b_{1}\right)+d_{G_{1}}\left(a_{2}\right) d_{G_{2}}\left(b_{2}\right)\right] .
\end{gathered}
$$


Again, we take

$$
\begin{gathered}
\sum_{b \in V\left(G_{2}\right)} \sum_{a_{1} a_{2} \notin E\left(G_{1}\right)}\left[\tau_{G_{1} \oplus G_{2}}\left(a_{1}, b\right)+\tau_{G_{1} \oplus G_{2}}\left(a_{2}, b\right)\right], \\
\text { Similarly }=2 n_{1} n_{2}^{2} \mu_{1}-2 n_{2} \mu_{1}-n_{2}^{2} \bar{M}_{1}\left(G_{1}\right)-4 n_{1} e_{2} \mu_{1}+2 e_{2} \bar{M}_{1}\left(G_{1}\right) .
\end{gathered}
$$

We further take (null case)

$$
N=\sum_{\substack{a_{1} a_{2} \notin E\left(G_{1}\right) \\ a_{1} \neq a_{2} \wedge a_{1} \sim a_{2}}} \sum_{\substack{b_{1} b_{2} \notin E\left(G_{2} \\ b_{1} \neq b_{2} \wedge b_{1} \uparrow b_{2}\right.}}\left[\tau_{G_{1} \oplus G_{2}}\left(a_{1}, b_{1}\right)+\tau_{G_{1} \oplus G_{2}}\left(a_{2}, b_{2}\right)\right]=0 .
$$

Furthermore, we take (also null case)

$$
N=\sum_{\substack{b_{1} b_{2} \notin E\left(G_{1}\right) \\ b_{1} \neq b_{2} \wedge b_{1} \sim b_{2}}} \sum_{\substack{a_{1} a_{2} \notin E\left(G_{2}\right) \\ a_{1} \neq a_{2} \wedge a_{1} \uparrow a_{2}}}\left[\tau_{G_{1} \oplus G_{2}}\left(a_{1}, b_{1}\right)+\tau_{G_{1} \oplus G_{2}}\left(a_{2}, b_{2}\right)\right]=0 .
$$

Consequently,

$$
\begin{aligned}
\bar{Z} C_{1}\left(G_{1} \oplus G_{2}\right)= & \left(2 e_{2}-n_{2}^{2}-2 n_{2} \mu_{2}\right) \bar{M}_{1}\left(G_{1}\right)+\left(2 e_{1}-n_{1}^{2}-2 n_{1} \mu_{1}\right) \bar{M}_{1}\left(G_{2}\right) \\
& +2 \mu_{1}\left(n_{1} n_{2}^{2}-n_{2}-2 n_{1} e_{2}\right)+2 \mu_{2}\left(n_{1}^{2} n_{2}-n_{1}-2 n_{2} e_{1}\right)+4 \mu_{1} \mu_{2}\left(n_{1} n_{2}-1\right) \\
& +2 \sum_{\substack{a_{1} a_{2} \notin E\left(G_{1}\right) \\
a_{1} \neq a_{2} \wedge a_{1} \sim a_{2}}} \sum_{b_{1} b_{2} \notin E\left(G_{2}\right)}\left[d_{G_{1}}\left(a_{1}\right) d_{G_{1}}\left(b_{1}\right)+b_{2}\right.
\end{aligned}
$$

\section{Analysis and Conclusions}

In this section, we compute the analysis for the first Zagreb connection index $\left(Z C_{1}\right)$ and coindex $\left(\bar{Z} C_{1}\right)$ of product on graphs such as tensor product, strong product, symmetric difference, and disjunction with the help of Tables 1-5 which are constructed by using numerical values of the aforesaid Zagreb index and coindex, respectively. The graphical depictions of the exact formulae and upper bounds for $Z C_{1}$ and $\bar{Z} C_{1}$ are also depicted in Figures 5-9. Moreover, in this section, for particular cases of main results, we compute all the results in the shape of upper bounds as $\left\{C_{3}, C_{4}\right\}$ are not free graphs and $G_{1}$ and $G_{2}$ are two undirected graphs.

4.1. Tensor Product. Let $P_{m}$ and $P_{n}$ be two particular alkanes called by paths, then the tensor product $\left(P_{m} \oslash P_{n}\right)$ is obtained by the product of $P_{m}$ and $P_{n}$. For $m=3$ and $n=3$, see Figure 10.
Using Theorem 1, the exact formulae for the first Zagreb connection index $\left(Z C_{1}\left(\theta_{1}\right)\right)$ and first Zagreb connection coindex $\left(\bar{Z} C_{1}\left(\psi_{1}\right)\right)$ of tensor product are obtained as follows:

(a) $Z C_{1}\left(P_{m} \oslash P_{n}\right) \leq 64 m n-138 m-138 n+292$

(b) $\bar{Z} C_{1}\left(P_{m} \oslash P_{n}\right) \leq 4 m n-4 m-6 n+6$

The upper bounds for the first Zagreb connection index $\left(Z C_{1}\left(\lambda_{1}\right)\right)$ of tensor product are obtained as follows [39]:

$$
Z C_{1}\left(P_{m} \oslash P_{n}\right) \leq 64 m n-56 m-124 n+100 .
$$

Table 1 and Figure 5 depict the numerical and graphical behaviours of the analysis between exact formulae and upper bounds for the first Zagreb connection index and coindex of tensor product by using values $m=n$.

4.2. Strong Product. Let $P_{m}$ and $P_{n}$ be two particular alkanes called by paths, then the strong product $\left(P_{m} \otimes P_{n}\right)$ is 
TABle 1: Analysis for index and coindex of exact formulae and upper bounds of $\theta_{1}=P_{m} \oslash P_{n}, \lambda_{1}=P_{m} \oslash P_{n}$, and $\psi_{1}=P_{m} \oslash P_{n}$, respectively.

\begin{tabular}{lccc}
\hline$(m=n)$ & $Z C_{1}\left(\theta_{1}\right)$ & $Z C_{1}\left(\lambda_{1}\right)$ & $\bar{Z} C_{1}\left(\psi_{1}\right)$ \\
\hline 1 & 80 & -16 & 0 \\
2 & -4 & -4 & 2 \\
3 & 40 & 136 & 12 \\
4 & 212 & 404 & 30 \\
5 & 512 & 800 & 56 \\
6 & 940 & 1324 & 90 \\
7 & 1496 & 1976 & 132 \\
8 & 2180 & 2756 & 182 \\
9 & 2992 & 3664 & 240 \\
10 & 3932 & 4700 & 306 \\
11 & 5000 & 5864 & 380 \\
12 & 6196 & 7156 & 462 \\
13 & 7520 & 8576 & 552 \\
14 & 8972 & 10124 & 650 \\
15 & 10552 & 11800 & 756 \\
\hline
\end{tabular}

TABle 2: Analysis for index and coindex of exact formulae and upper bounds of $\theta_{2}=P_{m} \oslash P_{n}, \lambda_{2}=P_{m} \oslash P_{n}$, and $\psi_{2}=P_{m} \oslash P_{n}$, respectively.

\begin{tabular}{lccc}
\hline$(m=n)$ & $Z C_{1}\left(\theta_{2}\right)$ & $Z C_{1}\left(\lambda_{2}\right)$ & $\bar{Z} C_{1}\left(\psi_{2}\right)$ \\
\hline 1 & 98 & -13 & 22 \\
2 & 56 & -40 & 88 \\
3 & 218 & 91 & 234 \\
4 & 584 & 644 & 460 \\
5 & 1154 & 1979 & 766 \\
6 & 1928 & 4552 & 1152 \\
7 & 2906 & 8915 & 1618 \\
8 & 4088 & 15716 & 2164 \\
9 & 5474 & 25699 & 2790 \\
10 & 7064 & 39704 & 3496 \\
11 & 8858 & 58667 & 4282 \\
12 & 10856 & 83620 & 5148 \\
13 & 13058 & 115691 & 6094 \\
14 & 15464 & 156104 & 7120 \\
15 & 18074 & 206179 & 8226 \\
\hline
\end{tabular}

obtained by the product of $P_{m}$ and $P_{n}$. For $m=4$ and $n=4$, see Figure 11.

Using Theorem 2, the exact formulae for the first Zagreb connection index $\left(Z C_{1}\left(\theta_{2}\right)\right)$ and first Zagreb connection coindex $\left(\bar{Z} C_{1}\left(\psi_{2}\right)\right)$ of strong product are obtained as follows:

(a) $Z C_{1}\left(P_{m} \otimes P_{n}\right) \leq 102 m n-174 m-174 n+344$

(b) $\bar{Z} C_{1}\left(P_{m} \otimes P_{n}\right) \leq 40 m n-26 m-28 n+36$

The upper bounds for first Zagreb connection index $\left(Z C_{1}\left(\lambda_{2}\right)\right)$ of strong product are obtained as follows [39]:

$$
\begin{aligned}
Z C_{1}\left(P_{m} \otimes P_{n}\right) \leq & 4 m n^{3}+16 m n^{2}-5 m n-4 m-12 n^{3} \\
- & 40 n^{2}+24 n+4 .
\end{aligned}
$$

Table 2 and Figure 6 depict the numerical and graphical behaviours of the analysis between exact formulae and upper
TABle 3: Analysis for index and coindex of exact formulae and upper bounds of $\theta_{3}=P_{m} \oslash P_{n}, \lambda_{3}=P_{m} \oslash P_{n}$, and $\psi_{3}=P_{m} \oslash P_{n}$, respectively.

\begin{tabular}{lccc}
\hline$(m=n)$ & $Z C_{1}\left(\theta_{3}\right)$ & $Z C_{1}\left(\lambda_{3}\right)$ & $\bar{Z} C_{1}\left(\psi_{3}\right)$ \\
\hline 1 & 12 & 33 & 0 \\
2 & 4 & 34 & 0 \\
3 & 116 & 153 & 44 \\
4 & 912 & 390 & 264 \\
5 & 4348 & 745 & 888 \\
6 & 15332 & 1218 & 2240 \\
7 & 44004 & 1809 & 4740 \\
8 & 108736 & 2518 & 8904 \\
9 & 239852 & 3345 & 15344 \\
10 & 484068 & 4290 & 24768 \\
11 & 909652 & 5353 & 37980 \\
12 & 1612304 & 6534 & 55880 \\
13 & 2721756 & 7833 & 79464 \\
14 & 4409092 & 9250 & 109824 \\
15 & 6894788 & 10785 & 148148 \\
\hline
\end{tabular}

TABle 4: Analysis for index and coindex of exact formulae and upper bounds of $\theta_{4}=P_{m} \oslash P_{n}, \lambda_{4}=P_{m} \oslash P_{n}$, and $\psi_{4}=P_{m} \oslash P_{n}$, respectively.

\begin{tabular}{lccc}
\hline$(m=n)$ & $Z C_{1}\left(\theta_{4}\right)$ & $Z C_{1}\left(\lambda_{4}\right)$ & $\bar{Z} C_{1}\left(\psi_{4}\right)$ \\
\hline 1 & 0 & 141 & 0 \\
2 & 0 & 40 & -2 \\
3 & 40 & 189 & 40 \\
4 & 492 & 588 & 378 \\
5 & 2928 & 1237 & 1456 \\
6 & 11680 & 2136 & 3910 \\
7 & 36120 & 3285 & 8568 \\
8 & 93660 & 4684 & 16450 \\
9 & 213472 & 6333 & 28768 \\
10 & 440928 & 8232 & 46926 \\
11 & 842760 & 10381 & 72520 \\
12 & 1512940 & 12780 & 107338 \\
13 & 2579280 & 15429 & 153360 \\
14 & 4210752 & 18328 & 212758 \\
15 & 6676153 & 21477 & 287896 \\
\hline
\end{tabular}

TABLE 5: Particular numeric values of obtained results for index and coindex.

\begin{tabular}{lccc}
\hline Product graphs & $Z C_{1}(\theta)$ & $Z C_{1}(\lambda)$ & $\bar{Z} C_{1}(\psi)$ \\
\hline$\left(P_{3} \oslash P_{3}\right)$ & 40 & 136 & 12 \\
$\left(P_{4} \otimes P_{4}\right)$ & 584 & 644 & 460 \\
$\left(P_{3} \ominus P_{3}\right)$ & 116 & 153 & 44 \\
$\left(P_{3} \oplus P_{3}\right)$ & 40 & 189 & 40 \\
\hline
\end{tabular}

bounds for the first Zagreb connection index and coindex of strong product by using values $m=n$.

4.3. Symmetric Difference. Let $P_{m}$ and $P_{n}$ be two particular alkanes called by paths, then the symmetric difference $\left(P_{m} \ominus P_{n}\right)$ is obtained by the product of $P_{m}$ and $P_{n}$. For $m=3$ and $n=3$, see Figure 12 . 


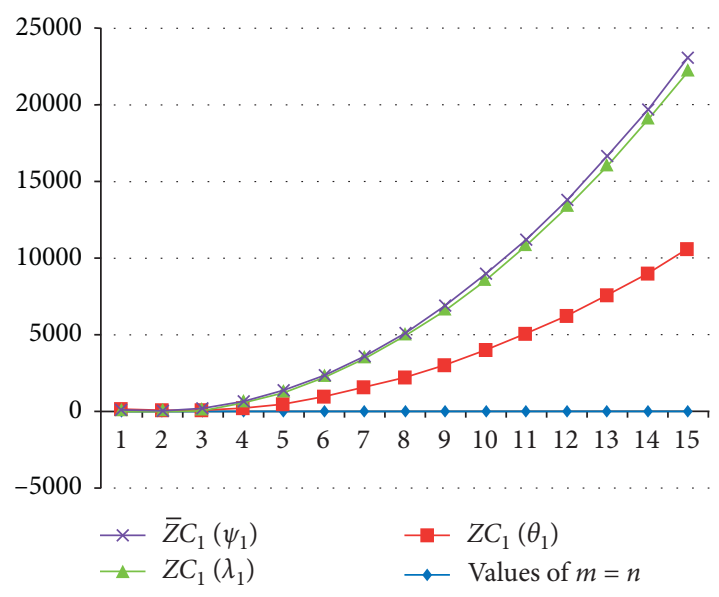

Figure 5: Analysis for index and coindex of tensor product with respect to Table 1 .

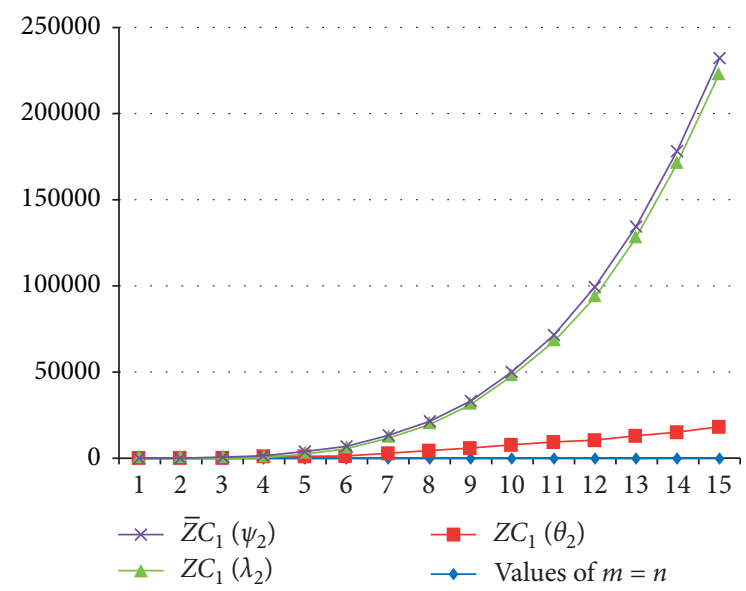

Figure 6: Analysis for index and coindex of strong product with respect to Table 2 .

Using Theorem 3, the exact formulae for the first Zagreb connection index $\left(Z C_{1}\left(\theta_{3}\right)\right)$ and first Zagreb connection

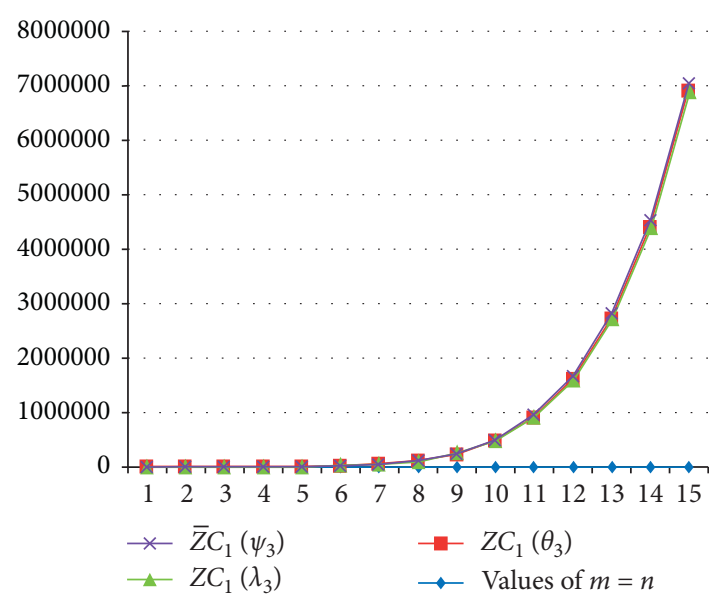

Figure 7: Analysis for index and coindex of symmetric difference with respect to Table 3 .

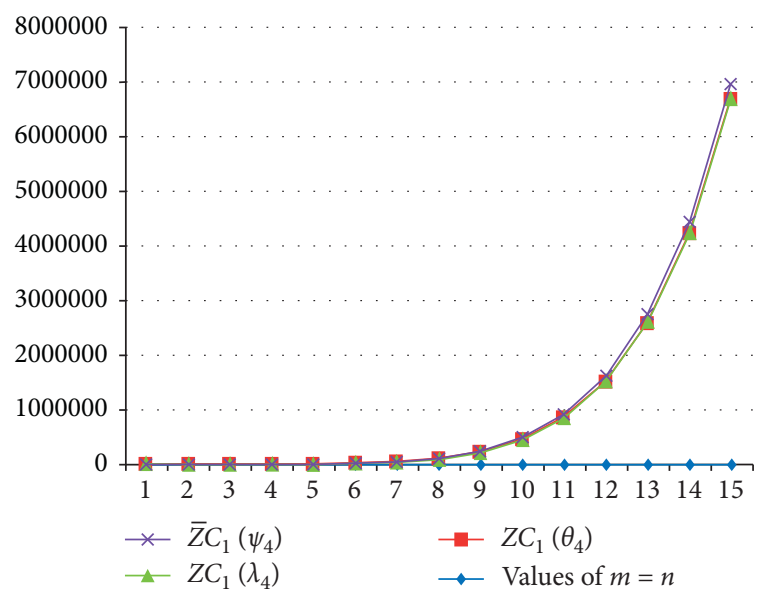

Figure 8: Analysis for index and coindex of disjunction with respect to Table 4 .

coindex $\left(\bar{Z} C_{1}\left(\psi_{3}\right)\right)$ of symmetric difference are obtained as follows:

$$
\begin{aligned}
Z C_{1}\left(P_{m} \ominus P_{n}\right) \leq & m^{3} n^{3}-4 m^{3} n^{2}+8 m^{3} n-6 m^{3}-4 m^{2} n^{3}+22 m^{2} n^{2}-52 m^{2} n+44 m^{2}+8 m n^{3}-52 m n^{2} \\
& +137 m n-128 m-6 n^{3}+44 n^{2}-128 n+128, \\
\bar{Z} C_{1}\left(P_{m} \ominus P_{n}\right) \leq & 4 m^{2} n^{2}-9 m^{2} n+5 m^{2}-9 m n^{2}+20 m n-12 m+5 n^{2}-12 n+8 .
\end{aligned}
$$

The upper bounds for first Zagreb connection index $\left(Z C_{1}\left(\lambda_{3}\right)\right)$ of symmetric difference are obtained as follows [40]:

$$
Z C_{1}\left(P_{m} \ominus P_{n}\right) \leq 59 m n-88 m-88 n+150 .
$$

Table 3 and Figure 7 depict the numerical and graphical behaviours of the analysis between exact formulae and upper bounds for first Zagreb connection index and coindex of symmetric difference by using values $m=n$.
4.4. Disjunction. Let $P_{m}$ and $P_{n}$ be two particular alkanes called by paths, then the disjunction $\left(P_{m} \oplus P_{n}\right)$ is obtained by the product of $P_{m}$ and $P_{n}$. For $m=3$ and $n=3$, see Figure 13.

Using Theorem 4, the exact formulae for the first Zagreb connection index $\left(Z C_{1}\left(\theta_{4}\right)\right)$ and first Zagreb connection coindex $\left(\bar{Z} C_{1}\left(\psi_{4}\right)\right)$ of disjunction are obtained as follows: 


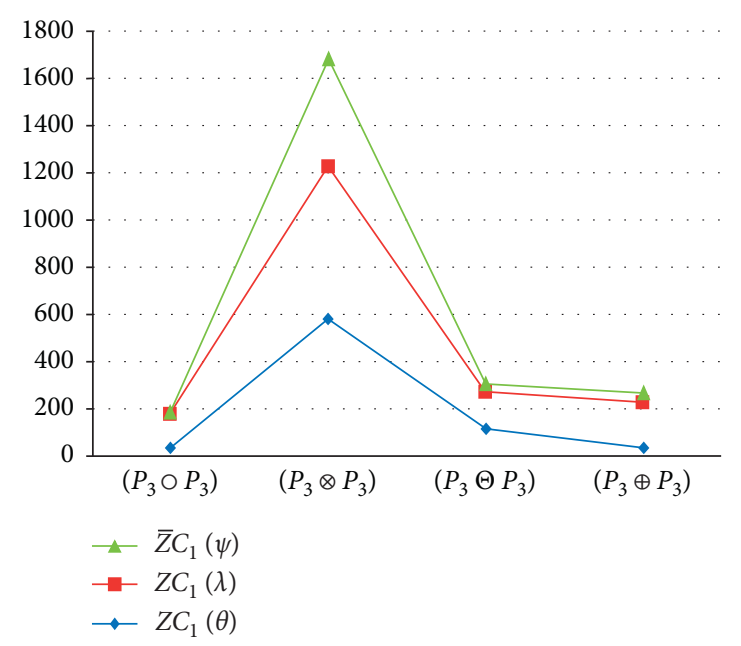

FIgURE 9: Analysis of obtained results for product on graphs with respect to Table 5.
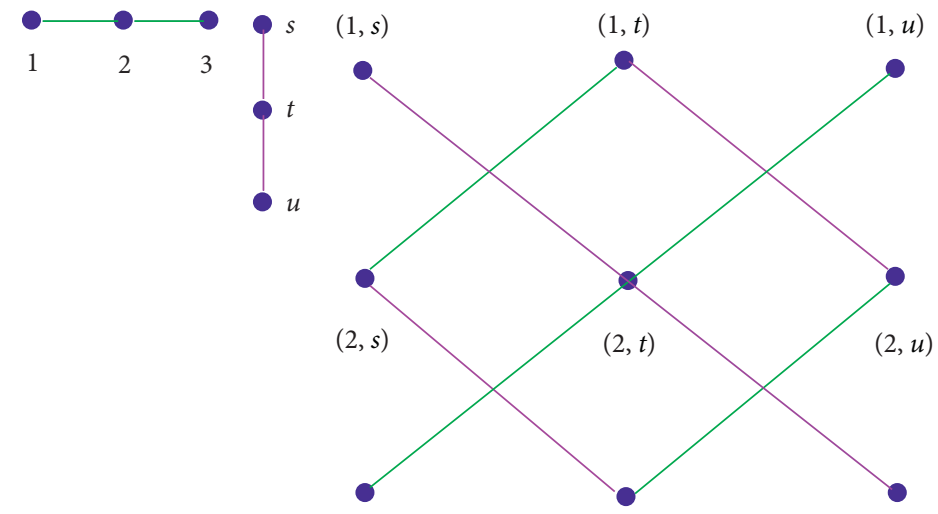

$(3, s)$

$(3, t)$

$(3, u)$

(a)

(b)

(c)

FIgURE 10 : (a) $G_{1} \cong P_{3}$ (b) $G_{2} \cong P_{3}$, and (c) tensor product $\left(P_{3} \oslash P_{3}\right)$.
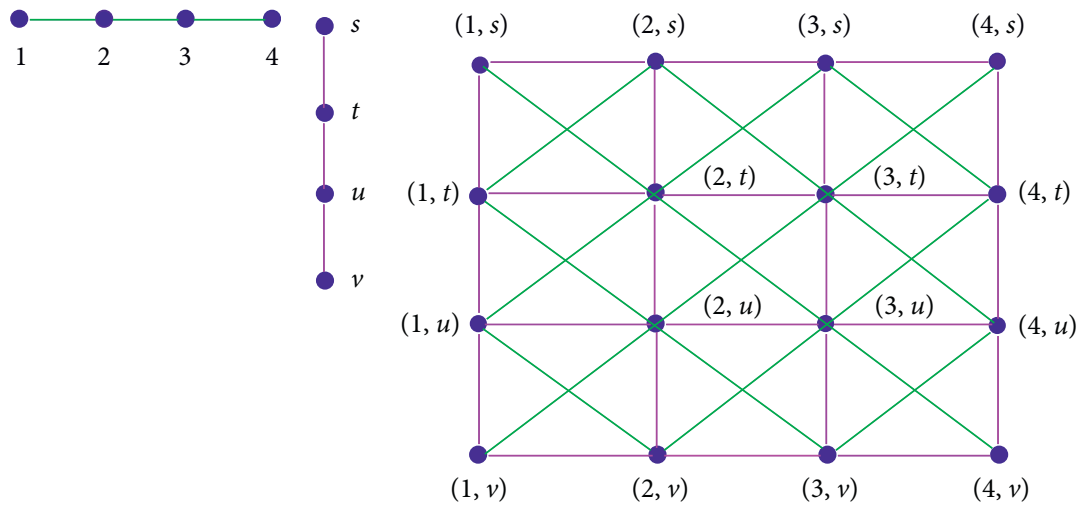

(a)

(b)

(c)

Figure 11: (a) $G_{1} \cong P_{4}$ (b) $G_{2} \cong P_{4}$, and (c) strong product $\left(P_{4} \otimes P_{4}\right)$. 

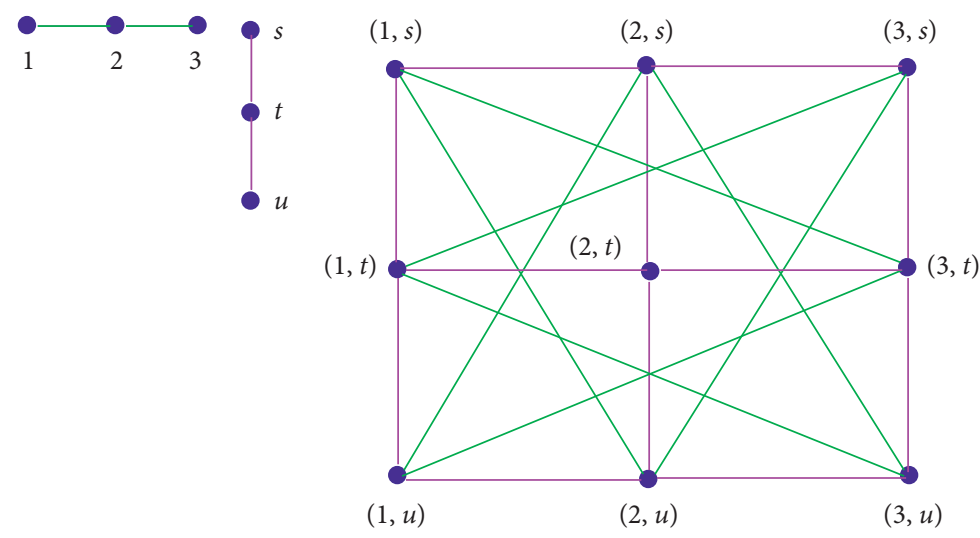

(a)

(b)

(c)

Figure 12: (a) $G_{1} \cong P_{3}$ (b) $G_{2} \cong P_{3}$, and (c) symmetric difference $\left(P_{3} \ominus P_{3}\right)$.
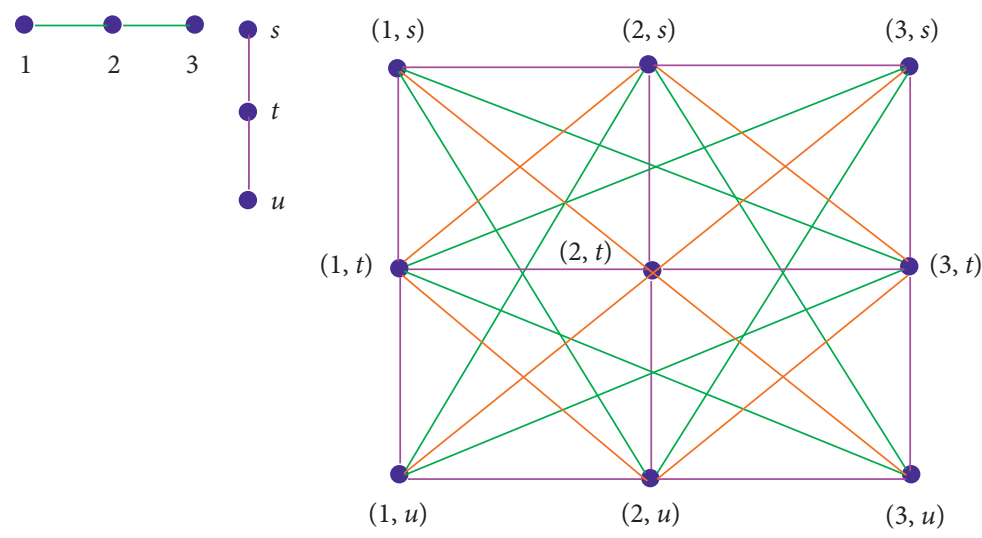

(a)

(b)

(c)

Figure 13: (a) $G_{1} \cong P_{3}$ (b) $G_{2} \cong P_{3}$, and (c) disjunction $\left(P_{3} \oplus P_{3}\right)$.

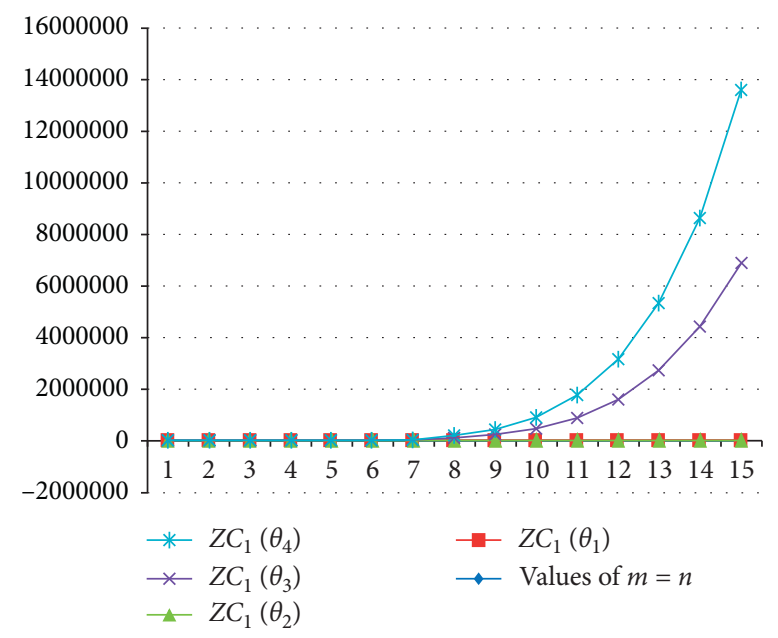

Figure 14: Analysis for $Z C_{1}(\theta)$ of exact formula. 


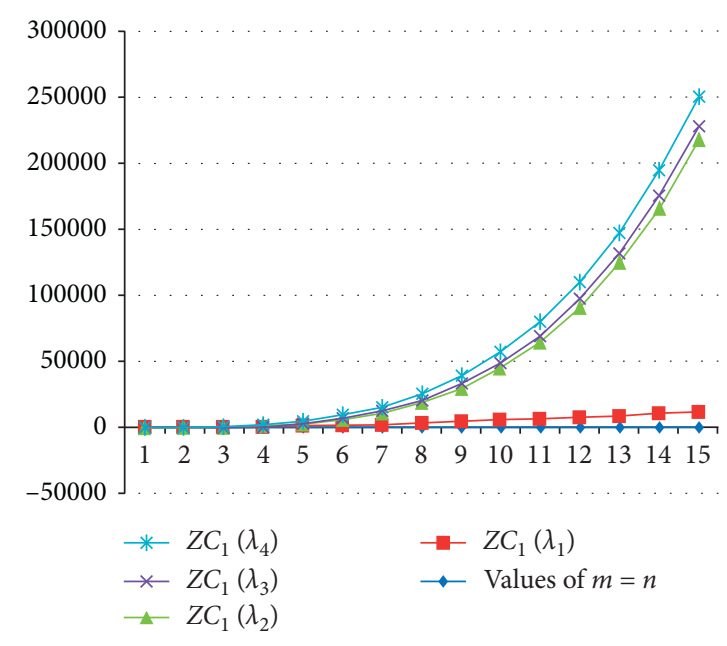

Figure 15: Analysis for $Z C_{1}(\lambda)$ of upper bound.

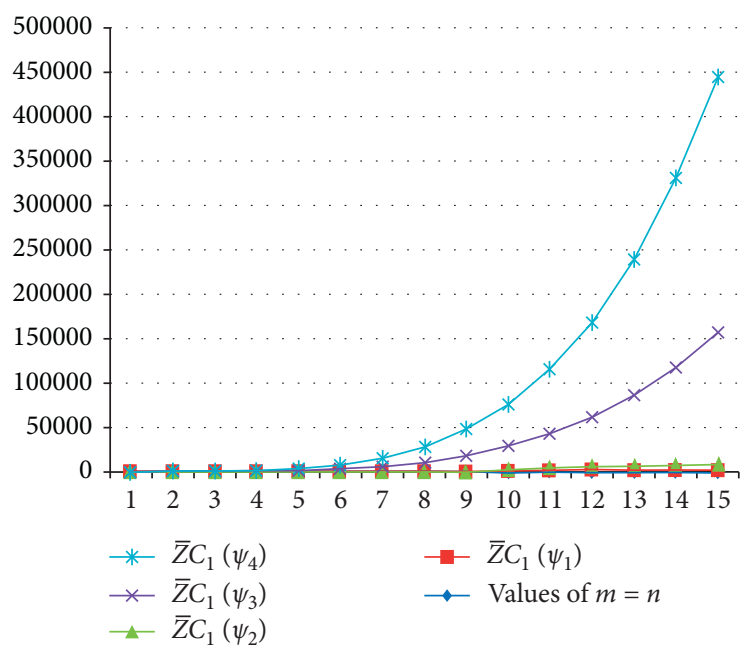

Figure 16: Analysis for $\bar{Z} C_{1}(\psi)$ of exact formula.

$$
\begin{aligned}
Z C_{1}\left(P_{m} \oplus P_{n}\right) \leq & m^{3} n^{3}-4 m^{3} n^{2}+8 m^{3} n-6 m^{3}-4 m^{2} n^{3}+14 m^{2} n^{2}-28 m^{2} n+20 m^{2}+8 m n^{3} \\
& -28 m n^{2}+41 m n-40 m-6 n^{3}+36 n^{2}-40 n+28, \\
\bar{Z} C_{1}\left(P_{m} \oslash P_{n}\right) \leq & 8 m^{2} n^{2}-19 m^{2} n+7 m^{2}-19 m n^{2}+36 m n-2 m+7 n^{2}-4 n-14 .
\end{aligned}
$$

The upper bounds for first Zagreb connection index $\left(Z C_{1}\left(\lambda_{4}\right)\right)$ of disjunction are obtained as follows [39]:

$$
Z C_{1}\left(P_{m} \oplus P_{n}\right) \leq 125 m n-238 m-238 n+492 .
$$

Table 4 and Figure 8 depict the numerical and graphical behaviours of the analysis between exact formulae and upper bounds for the first Zagreb connection index and coindex of disjunction by using values $m=n$.

Now, from Tables $1-5$ and Figures 5-9 and 14-16, we close our discussion with the following conclusions:

The behaviours of all the connection number-based

Zagreb index and coindex for operations on graphs such as tensor product, strong product, symmetric difference, and disjunction are increased in the following order, respectively, as $\bar{Z} C_{1}(\psi) \geq Z C_{1}(\lambda) \geq$ $Z C_{1}(\theta)$.

For increasing values of $m$ and $n$, the upper bound for the first Zagreb connection index of products on graphs are working rapidly than all the exact formula for the first Zagreb connection index, respectively.

In certain intervals of the values of $m$ and $n$, all the first Zagreb connection coindices attain the maximum values on increasing values of $m$ and $n$. In Figures 5-8, we analyse that the first Zagreb connection coindex 
attains more upper layer than other TIs in all the operations.

Table 5 and Figure 9 interpret the particular analysis of the obtained results for index and coindex on operations such as tensor product, strong product, symmetric difference, and disjunction. This particular analysis also concludes that the first Zagreb connection coindex attains more upper layer than other TIs in all the operations.

In particular, Figures 14-16 interpret the exact formula for the first Zagreb connection index, upper bound for the first Zagreb connection index, and exact formula for the first Zagreb connection coindex which are dominant on operations from tensor product to disjunction, respectively. In addition, we analyse that the first Zagreb connection coindex of operation disjunction has attained more upper layer than all the other operations for connection number-based index and coindex.

The investigation of these indices and coindices for the resultant graphs obtained from other operations of graphs (subtraction, switching, zig-zag product, addition, rooted product, modular product etc.) is still open.

\section{Data Availability}

All data used to support the findings of this study are included within the article. However, additional data will be made available from the corresponding author upon request.

\section{Conflicts of Interest}

The authors declare that they have no conflicts of interest.

\section{Acknowledgments}

This work was supported by the Humanities and Social Science Project of Anhui Provincial Education Department, Research on social crisis management in Anhui cities under the background of big data (subject no: SK2016A0233).

\section{References}

[1] R. Todeschini and V. Consonni, Molecular Descriptors for Chemoinformatics, Wiley-VCH Verlag GmbH, Weinheim, Germany, 2009.

[2] A. R. Matamala and E. Estrada, "Generalised topological indices: optimisation methodology and physico-chemical interpretation," Chemical Physics Letters, vol. 410, no. 4-6, pp. 343-347, 2005.

[3] H. Gonzalez-Diaz, S. Vilar, L. Santana, and E. Uriarte, "Medicinal chemistry and bioinformatics - current trends in drugs discovery with networks topological indices," Current Topics in Medicinal Chemistry, vol. 7, no. 10, pp. 1015-1029, 2007.

[4] G. Rücker and C. Rücker, "On topological indices, boiling points, and cycloalkanes," Journal of Chemical Information and Computer Sciences, vol. 39, no. 5, pp. 788-802, 1999.
[5] B. Furtula and I. Gutman, "A forgotten topological index," Journal of Mathematical Chemistry, vol. 53, no. 4, pp. 11841190, 2015.

[6] A. R. Ashrafi, T. Došlić, and A. Hamzeh, "The Zagreb coindices of graph operations," Discrete Applied Mathematics, vol. 158, no. 15, pp. 1571-1578, 2010.

[7] K. C. Das, A. Yurttas, M. Togan, A. S. Cevik, and I. N. Cangul, "The multiplicative Zagreb indices of graph operations," Journal of Inequalities and Applications, vol. 90, p. 14, 2013.

[8] K. Xu, K. C. Das, and K. Tang, "On the multiplicative Zagreb coindex of graphs," Opuscula Mathematica, vol. 33, no. 1, pp. 191-204, 2013.

[9] S. M. Hosamani and N. Trinajstić, "On reformulated zagreb coindices, research gate, T 09:07:00 UTC," 2015.

[10] K. Pattabiraman, S. Nagarajan, and M. Chendrasekharan, "Zagreb indices and coindices of product graphs," Journal of Progressive Research in Mathematics, vol. 10, pp. 80-91, 2015.

[11] B. Basavanagoud, I. Gutman, and C. S. Gali, "On second Zagreb index and coindex of some derived graphs," Kragujevac Journal of Science, vol. 37, pp. 113-121, 2015.

[12] N. De, S. M. A. Nayeem, and A. Pal, "The F-coindex of some graph operations," SpringerPlus, vol. 5, no. 221, p. 13, 2016.

[13] M. Veylaki, M. J. Nikmehr, and H. A. Tavallaee, "The third and hyper-Zagreb coindices of some graph operations," Journal of Applied Mathematics and Computing, vol. 50, no. 12, pp. 315-325, 2016.

[14] H. Hua and S. Zhang, "Relations between Zagreb coindices and some distance-based topological indices," MATCH Communications in Mathematical and in Computer Chemistry, vol. 68, pp. 199-208, 2012.

[15] M. Azari and A. Iranmanesh, "Chemical graphs constructed from rooted product and their Zagreb indices," MATCH Communications in Mathematical and in Computer Chemistry, vol. 70, pp. 901-919, 2013.

[16] N. De, A. Pal, and S. M. A. Nayeem, "On some bounds and exact formulae for connective eccentric indices of graphs under some graph operations," International Journal of Mathematical Combinatorics, vol. 2014, Article ID 579257, 5 pages, 2014.

[17] Z. Luo and J. Wu, "Zagreb eccentricity indices of the generalized Hierachical product graphs and their applications," Journal of Applied Mathematics, vol. 2014, Article ID 241712, 8 pages, 2014.

[18] W. Gao, M. K. Jamil, and M. R. Farahani, "The hyper-Zagreb index and some graph operations," Journal of Applied Mathematics and Computing, vol. 54, no. 1-2, pp. 263-275, 2017.

[19] I. Gutman and N. Trinajstić, "Graph theory and molecular orbitals. Total $\varphi$-electron energy of alternant hydrocarbons," Chemical Physics Letters, vol. 17, no. 4, pp. 535-538, 1972.

[20] I. Gutman, "Degree-based topological indices," Croatica Chemica Acta, vol. 86, no. 4, pp. 351-361, 2013.

[21] J.-B. Liu, S. Javed, M. Javaid, and K. Shabbir, "Computing first general Zagreb index of operations on graphs," IEEE Access, vol. 7, pp. 47494-47502, 2019.

[22] J.-B. Liu, M. Javaid, and H. M. Awais, "Computing Zagreb indices of the subdivision-related generalized operations of graphs," IEEE Access, vol. 7, pp. 105479-105488, 2019.

[23] X. Zhang, H. M. Awais, M. Javaid, and M. K. Siddiqui, "Multiplicative zagreb indices of molecular graphs," Journal of Chemistry, vol. 2019, pp. 1-19, 2019.

[24] H. M. Awais, M. Javaid, and M. Jamal, "Forgotten index of generalized F-sum graphs," Journal of Prime Research in Mathematics, vol. 15, pp. 115-128, 2019. 
[25] H. M. Awais, M. Javaid, and A. Raheem, "Hyper-Zagreb index of graphs based on generalized subdivision related operations," Punjab University Journal of Mathematics, vol. 52, no. 5, pp. 89-103, 2019.

[26] G. Hong, Z. Gu, M. Javaid, H. M. Awais, and M. K. Siddiqui, "Degree-based topological invariants of metal-organic networks," IEEE Access, vol. 8, pp. 68288-68300, 2020.

[27] H. M. Awais, M. Jamal, and M. Javaid, “Topological properties of metal-organic frameworks," Main Group Metal Chemistry, vol. 43, no. 1, pp. 67-76, 2020.

[28] H. M. Awais, M. Javaid, and A. Akbar, "First general Zagreb index of generalized F-sum graphs," Discrete Dynamics in Nature and Society, vol. 2020, Article ID 2954975, 2020.

[29] Y.-M. Chu, S. Javed, M. Javaid, and M. Kamran Siddiqui, "On bounds for topological descriptors of $\varphi$-sum graphs," Journal of Taibah University for Science, vol. 14, no. 1, pp. 1288-1301, 2020.

[30] A. Ali and N. Trinajstić, "A novel/old modification of the first Zagreb index," Molecular Informatics, vol. 37, pp. 1-7, 2018.

[31] U. Ali, M. Javaid, and A. M. Alanazi, "Computing analysis of connection-based indices and coindices for product of molecular networks," Symmetry, vol. 12, no. 1320, 23 pages, 2020.

[32] G. Ducoffe, R. Marinescu-Ghemeci, C. Obeja, A. Popa, and R. M. Tache, "Extremal graphs with respect to the modified first Zagreb connection Index," in Proceedings of the 16th cologne-twente workshop on graphs and combinatorial optimization, Paris, France, June 2018.

[33] Z. Shao, I. Gutman, Z. Li, S. Wang, and P. Wu, "Leap Zagreb indices of trees and unicyclic Graphs," Communications in Combinatorics and Optimization, vol. 3, pp. 179-194, 2018.

[34] A. M. Naji and N. D. Soner, "The first leap Zagreb index of some graph operations," International Journal of Applied Graph Theory, vol. 2, no. 1, pp. 7-18, 2018.

[35] I. Gutman, E. Milovanović, and I. Milovanović, "Beyond the Zagreb indices," AKCE International Journal of Graphs and Combinatorics, vol. 22, 2018, (In Press).

[36] J.-H. Tang, U. Ali, M. Javaid, and K. Shabbir, "Zagreb connection indices of subdivision and semi-total point operations on graphs," Journal of Chemistry, vol. 2019, Article ID 9846913, 14 pages, 2019.

[37] U. Ali, M. Javaid, and A. Kashif, "Modified Zagreb connection indices of the T-sum graphs," Main Group Metal Chemistry, vol. 43, no. 1, pp. 43-55, 2020.

[38] J. Cao, U. Ali, M. Javaid, and C. Huang, "Zagreb connection indices of molecular graphs based on operations," Complexity, vol. 2020, Article ID 7385682, 15 pages, 2020.

[39] U. Ali and M. Javaid, "Upper bounds of Zagreb connection indices of tensor and strong product on graphs," Punjab University Journal of Mathematics, vol. 52, no. 4, pp. 89-100, 2020.

[40] U. Ali and M. Javaid, "Zagreb connection indices of disjunction and symmetric difference operations on graphs," Journal of Prime Research in Mathematics, vol. 16, no. 2, pp. 1-15, 2020.

[41] D. Gong, Y. Han, and J. Sun, "A novel hybrid multi-objective artificial bee colony algorithm for blocking lot-streaming flow shop scheduling problems," Knowledge-Based Systems, vol. 16, no. 2, pp. 1-37, 2018.

[42] D. Gong, B. Xu, Y. Zhang, Y. Guo, and S. Yang, "A similaritybase cooperative co-evolutionary algorithm for dynamic interval multi-objective optimization problems," IEEE Ttansactions on Evolutionary Computation, vol. 17, 2019.

[43] J. Sun, D. Gong, and X. Sun, "Solving interval multi-objective optimization problems using evolutionary algorithms with preference polyhedron," in Proceedings of the Conference Paper in Information Sciences, Kuala Lumpur, Malaysia, November 2011.

[44] B. Xu, Y. Zhang, D. Gong, Y. Guo, and M. Rong, "Environment sensitivity-based cooperative co-evolutionary algorithms for dynamic multi-objective optimizatio," IEEE/ACM Transactions on Computational Biology and Bioinformatics, vol. 14, no. 1, 2020.

[45] F. Harary, Graph Theory, Addison-Wesley, Boston, MA, USA, 1994.

[46] W. Imrich and S. Klavzar, Product Graphs: Structure and Recognition, Wiley, New York, NY, USA, 2000.

[47] I. Gutman, B. Ruscic, N. Trinajstić, and C. F. Wilson, "Graph theory and molecular orbitals. XII. Acyclic polyenes," The Journal of Chemical Physics, vol. 62, no. 9, pp. 3399-3405, 1975.

[48] Klavzar, S. ar and I. Gutman, "Selected properties of the Schultz molecular topological index," Journal of Chemical Information and Modeling, vol. 36, pp. 1001-1003, 1996.

[49] U. Ali, M. Javaid, and Y.-M. Chu, "Computing relations between connection based modified indices and coindices for product of molecular-networks," Journal of Molecular Liquids, vol. 46, no. 1, 2021, (In Press).

[50] D. B. West, An Introduction to Graph Theory, Prentice-Hall, Upper Saddle River, NJ, USA, 1996. 